MATHEMATICS OF COMPUTATION

Volume 77 , Number 261, January 2008, Pages 41-70

S $0025-5718(07) 02018-2$

Article electronically published on May 14, 2007

\title{
ROBUST A-POSTERIORI ESTIMATOR FOR ADVECTION-DIFFUSION-REACTION PROBLEMS
}

\author{
GIANCARLO SANGALLI
}

\begin{abstract}
We propose an almost-robust residual-based a-posteriori estimator for the advection-diffusion-reaction model problem.

The theory is developed in the one-dimensional setting. The numerical error is measured with respect to a norm which was introduced by the author in 2005 and somehow plays the role that the energy norm has with respect to symmetric and coercive differential operators. In particular, the mentioned norm possesses features that allow us to obtain a meaningful a-posteriori estimator, robust up to a $\sqrt{\log (P e)}$ factor, where $P e$ is the global Péclet number of the problem. Various numerical tests are performed in one dimension, to confirm the theoretical results and show that the proposed estimator performs better than the usual one known in literature.

We also consider a possible two-dimensional extension of our result and only present a few basic numerical tests, indicating that the estimator seems to preserve the good features of the one-dimensional setting.
\end{abstract}

\section{INTRODUCTION}

In the last few decades adaptive finite element methods (FEM) have gained importance for the numerical solution of partial differential equations. A-posteriori error estimators are an essential ingredient for adaptivity. In fact, a-posteriori error estimators are computable quantities that provide information about the numerical error, so that they may be used for making judicious mesh modifications.

The mathematical a-posteriori error theory of elliptic problems is by now rather mature. Indeed, this has been a very active area of research, from the eighties [5] to the present day (e.g., the convergence of an adaptive algorithm has been proved recently in [15]); a reference to this topic is, for example, [2].

On the contrary, the theory for advection-dominated problems is more recent (starting with [11, 23]) and still under development. Important advances have been achieved, but the developed analysis is not fully satisfactory even for the simplest one-dimensional problem. In this paper, we aim to provide a new insight in the theory.

We will mainly work with the one-dimensional linear advection-diffusion-reaction model problem:

$$
\mathrm{\iota} u:=-\varepsilon u^{\prime \prime}+\beta u^{\prime}+\rho u=f \text { in }(0, L), \quad u(0)=u(L)=0,
$$

Received by the editor December 6, 2004 and, in revised form, November 29, 2006.

2000 Mathematics Subject Classification. Primary 65N30, 65G99.

The author was supported in part by the PRIN 2004 project of the Italian MIUR.

(C)2007 American Mathematical Society Reverts to public domain 28 years from publication 
where $f$ is the source term and $\varepsilon>0, \beta$ and $\rho \geq 0$ are the coefficients of the differential operator $\mathrm{E}$. To keep the exposition as simple as possible, we restrict to constant coefficients, but this is not necessary to the theory we present.

The goal here is to estimate the numerical error $\left\|u-u_{h}\right\|, u_{h}$ being a finite element approximation of the exact solution $u$, by a residual-based estimator $\eta$, that is, an easily-computable expression which depends only on the numerical residual $f-\mathrm{E} u_{h}$. The quality of an a-posteriori error estimator is often measured by its effectivity index $\eta /\left\|u-u_{h}\right\|$. We are interested in deriving a robust estimator, whose effectivity index is bounded from above and bounded away from zero uniformly with respect to $f$, the mesh-size $h$ and the operator coefficients $\varepsilon, \beta, \rho$.

The mathematical analysis of robustness of a-posteriori estimators for advectiondiffusion-reaction problems was first addressed in [23. The estimator proposed in 23. yields to upper and lower bound of the error measured in the energy norm

$$
\left\|u-u_{h}\right\|_{E}:=\left(\varepsilon\left|u-u_{h}\right|_{H_{0}^{1}}^{2}+\rho\left\|u-u_{h}\right\|_{L^{2}}^{2}\right)^{1 / 2} .
$$

These estimators are shown to be robust, but only when the element Péclet number $|\beta| h / \varepsilon$ is small, which is restrictive in the advection-dominated regime. Later extensions of this approach are 6, 12, 16, 18, 26.

More recent contributions are [18, 25]. There, robust estimators are obtained, but the numerical error is evaluated in an ad hoc norm which is, in our opinion, not the most appropriate for problem (1.1). It is clear that the choice of the norm to be used is a crucial point; on one hand, one would like $\left\|u-u_{h}\right\|$ to be a good indicator of the quality of the numerical approximation (for example, in view of adaptivity), and on the other hand, the possibility and difficulties in deriving a-posteriori error estimates depend on the norm one selects.

In this paper, we first discuss the choice of a suitable norm for measuring the numerical error. Our choice is based on the results of [21, where a norm, having for (1.1) the role that the energy norm (1.2) has in the symmetric case $(\beta=0)$, is constructed. The norm is denoted as $\|\cdot\|_{V}$ in the present paper, and has a structure like

$$
\|\cdot\|_{V} \approx\left(\varepsilon|\cdot|_{H_{0}^{1}}^{2}+\left|\beta\left\|\cdot|\cdot|_{1 / 2}^{2}+\rho\right\| \cdot \|_{L^{2}}^{2}\right)^{1 / 2},\right.
$$

where $|\cdot|_{1 / 2}$ is a seminorm of order $1 / 2$. Then, we address the construction of an a-posteriori estimator for $\left\|u-u_{h}\right\|_{V}$. The main tools for achieving this goal are some results of [21] and the techniques of 23]. The proposed estimator is shown to be almost-robust. We mean, roughly speaking, that it is robust up to a factor which is only logarithmic with respect to the global Péctlet number $P e=|\beta| L / \varepsilon$, for large $P e$.

Our results have some similarities to those obtained in 7]; there, the authors estimate the numerical error in a norm which has a structure similar to (1.3), but they address wavelet numerical methods and use wavelet techniques.

The theory of residual-based a-posteriori estimates can be linked to the multiscale approach. We do not follow this point of view in the paper, and just refer the interested reader to $3,4,10,17$.

Here, the theoretical analysis and the general discussion is restricted to onedimensional model problem (1.1); we wish to stress that, in spite of its simplicity, the a-posteriori analysis of (1.1) is harder than it might seem at first. However, we also test a natural extension to two dimensions of our estimator, and, though we 
do not give any mathematical justification, the numerical experiments show that the estimator is still robust, at least in the model cases considered. Therefore, we hope that the paper can give some insight for dealing with the multi-dimensional case, surely more difficult from the theoretical standpoint.

The outline of the paper is as follows. The first part, $\sqrt{2}$, is devoted to the one-dimensional problem. In $\$ 2.1$ we review some of the main ideas proposed in the literature for the construction of residual-based a-posteriori estimators, and we identify the difficulties encountered when trying to apply the general elliptic theory to advection-dominated problems. In 2.2 we recall the construction and some properties of the norm $\|\cdot\|_{V}$. Next, \$2.3 contains some technical lemmas required for the analysis. The construction of the a-posteriori estimator is given in 2.4 In $\$ 2.5$ we present a variety of one-dimensional numerical tests that support the theory. In the second part, \$3, we present two tests of the two-dimensional extension of the estimator. Finally, we draw the conclusions in 44

\section{TheOrY AND NUMERICAL TESTS IN ONE-DIMENSION}

2.1. Preliminaries on residual-based estimators. Let $\Omega$ denote the interval $(0, L)$. We denote by $L^{2} \equiv L^{2}(\Omega)$ the usual Lebesgue space endowed with the norm $\|\cdot\|_{L^{2}}$, while $H^{1} \equiv H^{1}(\Omega)$ is the usual Sobolev space endowed with the norm $\|\cdot\|_{H^{1}}$ and seminorm $|\cdot|_{H^{1}} ; H_{0}^{1}$ denotes the subspace of functions vanishing at 0 and $L$, endowed with the norm $|\cdot|_{H^{1}}$; finally $H^{-1} \equiv H^{-1}(\Omega):=\left(H_{0}^{1}\right)^{*}$ denotes the dual space of $H_{0}^{1}$ endowed with the dual norm $\|\cdot\|_{H^{-1}} ;\langle\cdot, \cdot\rangle \equiv_{H^{-1}}\langle\cdot, \cdot\rangle_{H_{0}^{1}}$ is the usual pairing between $H^{-1}$ and $H_{0}^{1}$. We shall make use of the $1 / 2$-order spaces $H^{1 / 2} \equiv H^{1 / 2}(\Omega)$ and $H_{00}^{1 / 2} \equiv H_{00}^{1 / 2}(\Omega)$, endowed with their standard norms and seminorms (see 13 fore more details).

Let $\left\{\mathcal{T}_{h}\right\}_{h}$ be a family of subdivisions of the domain $\Omega$ into subintervals $T$. The family $\left\{\mathcal{T}_{h}\right\}_{h}$ is assumed to be locally quasi uniform, that is, the ratio of the sizes of two adjacent elements of $\mathcal{T}_{h}$ is bounded uniformly with respect to the family index $h$. We denote by $T^{-}$and $T^{+}$the first and the last element of $\mathcal{T}_{h}$, respectively, and by $\mathcal{T}_{h}^{\circ}$ the set of all internal elements, that is, $\mathcal{T}_{h}^{\circ}:=\mathcal{T}_{h} \backslash\left\{T^{-}, T^{+}\right\}$. Let $\mathcal{Z}_{h}$ be the set of all the nodes of $\mathcal{T}_{h}$ and let $\mathcal{Z}_{h}^{\circ}$ be the subset of the internal nodes, that is, $\mathcal{Z}_{h}^{\circ}=\mathcal{Z}_{h} \backslash\{0, L\}$. Let $V_{h} \subset H^{1}(\Omega)$ be the usual $p$-degree finite element space

$$
V_{h}:=\left\{v \in H^{1}(\Omega) \mid v_{\mid T} \text { is polynomial of degree } \leq p, \forall T \in \mathcal{T}_{h}\right\},
$$

and $V_{h, 0}:=V_{h} \cap H_{0}^{1}(\Omega)$. We denote by $h_{T}$ the length of $T \in \mathcal{T}_{h}$ and, for a node $z$ which is shared between $T_{1}$ and $T_{2}, h_{z}$ is the minimum of $h_{T_{1}}$ and $h_{T_{2}}$.

In what follows, $C$ denotes a constant, possibly different at any occurrence, but independent of the mesh-size, the domain length $L$ and the coefficients $\varepsilon, \beta, \rho$. Moreover, $a \preceq b$ means $a \leq C b$, and $a \simeq b$ means $a \preceq b$ and $b \preceq a$ at the same time.

Let $a(\cdot, \cdot)$ be the bilinear form associated with the operator $\mathrm{E}$, that is,

$$
a(w, v):=\varepsilon \int_{0}^{L} w^{\prime} v^{\prime}+\beta \int_{0}^{L} w^{\prime} v+\rho \int_{0}^{L} w v, \quad \forall w, v \in H_{0}^{1} .
$$

Let $u_{h} \in V_{h, 0}$ be the numerical solution of (1.1) either by a plain Galerkin formulation,

$$
a\left(u_{h}, v_{h}\right)=\left\langle f, v_{h}\right\rangle, \quad \forall v_{h} \in V_{h, 0},
$$


or by a (one-dimensional) SUPG formulation (see [9])

$$
a\left(u_{h}, v_{h}\right)+\sum_{T \in \mathcal{T}_{h}} \tau_{T} \int_{T} \mathrm{Ł} u_{h} \beta v_{h}^{\prime}=\left\langle f, v_{h}\right\rangle+\sum_{T \in \mathcal{T}_{h}} \tau_{T} \int_{T} f \beta v_{h}^{\prime}, \quad \forall v_{h} \in V_{h, 0}
$$

where

$$
0 \leq \tau_{T} \preceq h_{T} /|\beta|, \quad \forall T \in \mathcal{T}_{h},
$$

which actually includes (2.1). We also assume, for the sake of simplicity, that $f$ is piecewise polynomial of degree at most $k$ on $\mathcal{T}_{h}$.

Consider first the simplest case when $\varepsilon=1$ and $\beta=\rho=0$, that is, $\mathrm{\iota} w=-w^{\prime \prime}$ is the one-dimensional Laplace operator. The construction and analysis of the residual-based estimator relies on two key steps,

$$
\begin{aligned}
\left\|u-u_{h}\right\|_{H_{0}^{1}} & \stackrel{1}{\simeq}\left\|f-\mathrm{E} u_{h}\right\|_{H^{-1}} \\
& \stackrel{2}{\simeq}\left(\sum_{T \in \mathcal{T}_{h}} h_{T}^{2}\left\|f-\mathrm{E} u_{h}\right\|_{L^{2}(T)}^{2}+\sum_{z \in \mathcal{Z}_{h}^{\circ}} h_{z}\left(\left[u_{h}^{\prime}\right](z)\right)^{2}\right)^{1 / 2},
\end{aligned}
$$

where $\left[u_{h}^{\prime}\right](z):=\lim _{\delta \rightarrow 0^{+}} u_{h}^{\prime}(z+\delta)-u_{h}^{\prime}(z-\delta)$. Step 1 is due to the fact that $\mathrm{\textrm {E }}$ is an isometry from $H_{0}^{1}$ into $H^{-1}$; precisely $\|w\|_{H_{0}^{1}} \equiv|w|_{H^{1}}=\|\mathrm{\textrm {E } w}\|_{H^{-1}}$, for all $w \in H_{0}^{1}$. Step 2 is due to the properties of the residual $f-\mathrm{\iota} u_{h}$; in particular,

$$
\left\|f-\mathrm{\iota} u_{h}\right\|_{H^{-1}} \preceq\left(\sum_{T \in \mathcal{T}_{h}} h_{T}^{2}\left\|f-\mathrm{\iota} u_{h}\right\|_{L^{2}(T)}^{2}+\sum_{z \in \mathcal{Z}_{h}^{\circ}} h_{z}\left(\left[u_{h}^{\prime}\right](z)\right)^{2}\right)^{1 / 2}
$$

is based on the consistency of the numerical scheme, while

$$
\left(\sum_{T \in \mathcal{T}_{h}} h_{T}^{2}\left\|f-\mathrm{E} u_{h}\right\|_{L^{2}(T)}^{2}+\sum_{z \in \mathcal{Z}_{h}^{\circ}} h_{z}\left(\left[u_{h}^{\prime}\right](z)\right)^{2}\right)^{1 / 2} \preceq\left\|f-\mathrm{L} u_{h}\right\|_{H^{-1}}
$$

relies on the fact that $f-\mathrm{E} u_{h}$ is discrete, $f$ being piecewise polynomia 1 .

Observe that the residual $f-\mathrm{E} u_{h} \in H^{-1}$ is the sum of the smooth part, which is its restriction inside the elements $T \in \mathcal{T}_{h}$, and the distributional part concentrated on the nodes $z \in \mathcal{Z}_{h}^{\circ}$; these two components lead to the two terms of the estimator stated in (2.4).

Steps 1 and 2 , emphasized in (2.4), are general for a-posteriori estimates of this kind. In the first step we express the numerical error in terms of the residual $f-\mathrm{E} u$ : the residual is computable, but we have to evaluate a dual norm of it, typically. The second step consists of finding a computable approximation of this dual norm. The first step relies on the properties of the operator $\mathrm{E}$ and the norms involved, the second step is based on the structure of the residual, as discussed above.

\footnotetext{
${ }^{1}$ If $f$ is not piecewise polynomial, one can use a discrete approximation of $f$ and reason as before; another term, which depends on the difference between $f$ and its approximation, will appear in (2.5)-2.6); we refer to 15] for further details.
} 
We consider now the (symmetric) reaction-diffusion operator $\mathrm{\iota} w=-\varepsilon w^{\prime \prime}+\rho w$, and reason as above (see also [24]): step 1 requires the so-called energy norm

$$
\|v\|_{E}:=\left(\varepsilon|v|_{H_{0}^{1}}^{2}+\rho\|v\|_{L^{2}}^{2}\right)^{1 / 2}, \quad \forall v \in H_{0}^{1},
$$

and the corresponding dual energy norm

$$
\|w\|_{E^{*}}:=\sup _{v \in H_{0}^{1}} \frac{\langle w, v\rangle}{\|v\|_{E}}, \quad \forall w \in H_{0}^{1},
$$

which give

$$
\left\|u-u_{h}\right\|_{E}=\left\|f-\mathrm{E} u_{h}\right\|_{E^{*}}
$$

Step 2 is performed exploiting the structure of the residual, more or less as in (2.5) -(2.6). The final estimate, for a piecewise polynomial $f$, is

$$
\begin{aligned}
&\left\|u-u_{h}\right\|_{E} \stackrel{1}{\simeq}\left\|f-\mathrm{L} u_{h}\right\|_{E^{*}} \\
& \stackrel{2}{\simeq}\left(\sum_{T \in \mathcal{T}_{h}} \min \left\{\rho^{-1}, \varepsilon^{-1} h_{T}^{2}\right\}\left\|f-\mathrm{L} u_{h}\right\|_{L^{2}(T)}^{2}\right. \\
&\left.\quad+\sum_{z \in \mathcal{Z}_{h}^{\circ}} \min \left\{\rho^{-1 / 2} \varepsilon^{-1 / 2}, \varepsilon^{-1} h_{z}\right\} \varepsilon\left(\left[u_{h}^{\prime}\right](z)\right)^{2}\right)^{1 / 2} \\
&=: \widehat{\eta}_{E} .
\end{aligned}
$$

Details on (2.9) are omitted, since it is a particular case of what is proved in 2.4 .

Consider now the full advection-diffusion-reaction case, that is, $\mathrm{E} w=-\varepsilon w^{\prime \prime}+$ $\beta w^{\prime}+\rho w$. First, try to reason as in (2.9), still looking for an estimator of $\left\|u-u_{h}\right\|_{E}=$ $\left(\varepsilon\left|u-u_{h}\right|_{H_{0}^{1}}^{2}+\rho\left\|u-u_{h}\right\|_{L^{2}}^{2}\right)^{1 / 2}$. In fact, the coercivity of $\mathrm{\complement}$ (i.e., $\langle\mathrm{E} v, v\rangle=\|v\|_{E}^{2}$ ) yields $\|v\|_{E} \leq\|\mathrm{\textrm {L } v}\|_{E^{*}}$ but, due to the presence of the advective term, $\|\mathrm{E} v\|_{E^{*}} \npreceq$ $\|v\|_{E}$ in this case. In other words, step 1 of (2.9) now reduces to a bound only from above for the error. However, we still have step 2 of (2.9), which is only due to the properties of the residual. Summarizing, we obtain an error bound from above for the error $\left\|u-u_{h}\right\|_{E}$, by the estimator $\widehat{\eta}_{E}$ defined in (2.9), as in [23]:

$$
\left\|u-u_{h}\right\|_{E} \frac{1}{\leq}\left\|f-\mathrm{E} u_{h}\right\|_{E^{*}} \stackrel{2}{\simeq} \widehat{\eta}_{E} .
$$

In many situations, $\widehat{\eta}_{E}$ overestimates the error $\left\|u-u_{h}\right\|_{E}$, as we will see in 2.5 .

It is clear that the loss of robustness in the estimate (2.10) occurs in step 1 . Indeed, it holds (see [21, Lemma 1])

$$
\|v\|_{E}+\left\|\beta v^{\prime}\right\|_{E^{*}} \simeq\|\mathrm{L} v\|_{E^{*}}, \quad \forall v \in H_{0}^{1},
$$

and robustness of the a-posteriori estimate is recovered if we replace $\left\|u-u_{h}\right\|_{E}$ by $\left\|u-u_{h}\right\|_{E}+\left\|\beta\left(u^{\prime}-u_{h}^{\prime}\right)\right\|_{E^{*}}:$

$$
\left\|u-u_{h}\right\|_{E}+\left\|\beta\left(u^{\prime}-u_{h}^{\prime}\right)\right\|_{E^{*}} \stackrel{1}{\simeq}\left\|f-\mathrm{L} u_{h}\right\|_{E^{*}} \stackrel{2}{\simeq} \widehat{\eta}_{E} .
$$




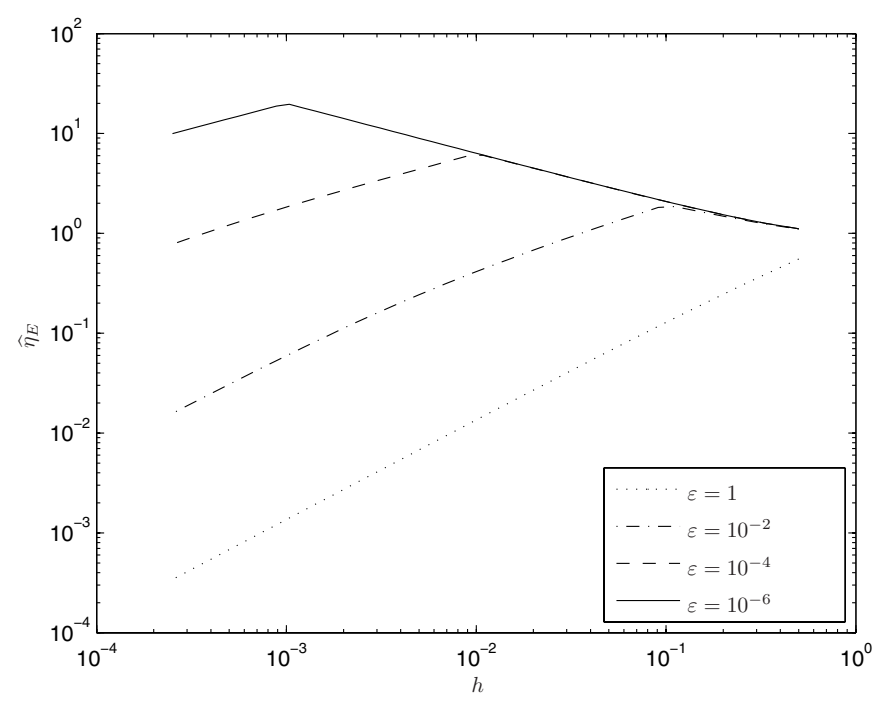

Figure 1. Behavior of $\widehat{\eta}_{E}$ vs. $h$, when $\beta=\rho=L=1$

In other words, $\widehat{\eta}_{E}$ is a robust estimator for $\left\|u-u_{h}\right\|_{E}+\left\|\beta\left(u^{\prime}-u_{h}^{\prime}\right)\right\|_{E^{*}}$, and not for $\left\|u-u_{h}\right\|_{E}$. This is the result of [18] and [25] ([18] is restricted to the advectiondiffusion case, that is, $\rho=0$ and deals with the Residual-free Bubbles FEM [8], but the analysis also applies to the plain Galerkin or SUPG FEM's).

Even though the a-posteriori estimate (2.12) is robust, we believe $\left\|u-u_{h}\right\|_{E}+$ $\left\|\beta\left(u^{\prime}-u_{h}^{\prime}\right)\right\|_{E^{*}}$ is not an appropriate indicator of the quality of the numerical approximation, when advection dominates. This opinion is supported by the following example. We take $\beta=\rho=1, L=1$ and $f=1$. When $\varepsilon$ is small, the exact solution $u$ has a boundary layer at $x=1$. We consider as a discrete solution $u_{h}$ the one given by the SUPG scheme (2.2) with (2.91), which is monotone and nodally accurate in this case. In Figure 1, we plot $\widehat{\eta}_{E}$ versus the mesh-size $h$ (uniform meshes are considered). We see that, when $\varepsilon \preceq h^{2} \rho$, refining the mesh results in an increase of $\widehat{\eta}_{E}$ and therefore, by virtue of (2.12), of $\left\|u-u_{h}\right\|_{E}+\left\|\beta\left(u^{\prime}-u_{h}^{\prime}\right)\right\|_{E^{*}}$. Precisely, $\widehat{\eta}_{E}$ is proportional to $h^{-1 / 2}$ in this region and reaches a maximum value proportional to $\varepsilon^{-1 / 4}$. This behavior makes it difficult to handle an adaptive mesh refinement.

In [21, we introduced, for general coercive and non-symmetric problems, a norm $\|\cdot\|_{V}$ which plays the same role of the energy norm for symmetric operators. Roughly speaking, this norm looks like $\left(\varepsilon|\cdot|_{H_{0}^{1}}^{2}+|\beta||\cdot|_{1 / 2}^{2}+\rho\|\cdot\|_{L^{2}}^{2}\right)^{1 / 2}$, where $|\cdot|_{1 / 2}$ is a non-standard seminorm of order $1 / 2$. The key property of $\|\cdot\|_{V}$ is stated in [21, Corollary 1]:

$$
\|v\|_{V} \simeq\|\mathrm{L} v\|_{V^{*}}, \quad \forall v \in H_{0}^{1} .
$$

The construction of such a norm is addressed in the next section. For what concerns the a-posteriori estimation, (2.13) can be used to perform the analogous of step 1 in (2.9):

$$
\left\|u-u_{h}\right\|_{V} \stackrel{1}{\simeq}\left\|f-\mathrm{E} u_{h}\right\|_{V^{*}}
$$


Next, following this path, we shall construct an estimator $\widehat{\eta}_{V}$ for $\left\|f-\mathrm{E} u_{h}\right\|_{V^{*}}$ which is almost-robust, that is, robust up to a factor $(\log (P e))^{1 / 2}$ for large Péclet numbers $P e:=|\beta| L / \varepsilon$. For practical purposes, the loss of robustness is weak (see the tests in $\$ 2.5$.

The ideas discussed above can be extended straightforwardly in multi-dimensions, with the exception of the construction of the a-posteriori estimator for $\left\|f-\mathrm{E} u_{h}\right\|_{V^{*}}$.

2.2. Construction and properties of the norm $\|\cdot\|_{V}$. We first recall some results of [21, specializing them for the one-dimensional operator $\mathrm{L} w=-\varepsilon w^{\prime \prime}+$ $\beta w^{\prime}+\rho w$. We introduce the sets $A_{0}=A_{1}=V=H_{0}^{1}(\Omega)$, which are Hilbert spaces endowed with the norms

$$
\begin{aligned}
& \|v\|_{A_{0}}:=\|v\|_{E}, \\
& \|v\|_{A_{1}}:=\left(\|v\|_{E}^{2}+\left\|\beta v^{\prime}\right\|_{E^{*}}^{2}\right)^{1 / 2},
\end{aligned}
$$

and, making use of the the interpolation theory of function spaces,

$$
\|v\|_{V}:=\|v\|_{\left(A_{0}, A_{1}\right)_{1 / 2,2}} .
$$

For the reader's convenience, we recall a possible definition of interpolated norm: given $0<\theta<1$ and $1 \leq p<+\infty$ we set

$$
\|v\|_{\left(A_{0}, A_{1}\right)_{\theta, p}}:=\left[\int_{0}^{+\infty} \inf _{\substack{v_{0} \in A_{0}, v_{1} \in A_{1}, v_{0}+v_{1}=v}}\left(t^{-2 \theta}\left\|v_{0}\right\|_{A_{0}}^{2}+t^{2-2 \theta}\left\|v_{1}\right\|_{A_{1}}^{2}\right)^{\frac{p}{2}} \frac{d t}{t}\right]^{\frac{1}{p}},
$$

while, for $p=\infty$,

$$
\|v\|_{\left(A_{0}, A_{1}\right)_{\theta, \infty}}:=\sup _{0<t<+\infty} \inf _{\substack{v_{0} \in A_{0}, v_{1} \in A_{1}, v_{0}+v_{1}=v}}\left(t^{-\theta}\left\|v_{0}\right\|_{A_{0}}+t^{1-\theta}\left\|v_{1}\right\|_{A_{1}}\right) .
$$

These are classical definitions, according to the K-method (see, for example, [22, §1.3.2], noting that (2.17) is modified to make (2.16) an Hilbertian norm).

The next result is needed for the construction of the a-posteriori estimator.

Lemma 2.1. For $T=T^{-}$or $T=T^{+}$(i.e., for the first or the last element of $\mathcal{T}_{h}$ ) we have

$$
|\beta|^{1 / 2} h_{T}^{-1 / 2}\|v\|_{L^{2}(T)} \preceq\left(1+\log ^{+}(P e)\right)^{1 / 2}\|v\|_{V}, \quad \forall v \in H_{0}^{1} .
$$

Moreover,

$$
|\beta|^{1 / 2} L^{-1 / 2}\|v\|_{L^{2}} \preceq\left(1+\log ^{+}(P e)\right)^{1 / 2}\|v\|_{V}, \quad \forall v \in H_{0}^{1} .
$$

Above, we have denoted by $\log ^{+}(\cdot)$ the positive part of the logarithm, that is, $\max \{\log (\cdot), 0\}$. The proof of Lemma 2.1 is postponed at the end of this section.

Continuity and infsup conditions with respect to $\|\cdot\|_{V}$ are stated in [21, Lemma 2]:

$$
\begin{aligned}
\text { continuity: } & \langle\mathrm{E} w, v\rangle \leq C_{\text {cont }}\|w\|_{V}\|v\|_{V}, \quad \forall v, w \in H_{0}^{1}, \\
\text { infsup: } & \inf _{w \in H_{0}^{1}} \sup _{v \in H_{0}^{1}} \frac{\langle\mathrm{L} w, v\rangle}{\|w\|_{V}\|v\|_{V}} \geq C_{\text {infsup }}>0 ;
\end{aligned}
$$

the constants $C_{\text {cont }}$ and $C_{\text {infsup }}$ are independent of the coefficients of $\mathrm{E}$, which gives (2.13). Notice that in (2.21) the norm $\|\cdot\|_{V}$ acts both on test and trial functions. 
For this reason, (2.21) seem natural extensions of the usual coercivity and continuity conditions for symmetric operators (i.e., $\beta=0$ ), where the energy norm appears.

Insights on the structure of $\|\cdot\|_{V}$ are given in [21, Lemma 3]. For this, we need the two spaces $C_{0}:=\left\{v \in L^{2} \mid \int_{\Omega} v=0\right\}$ and $C_{1}=H^{-1}$, endowed with the norms

$$
\begin{array}{ll}
\|v\|_{C_{0}}:=\left\|\beta^{-1} \nu\right\|_{E}, & \forall v \in C_{0}, \text { and } \nu(x)=\int_{0}^{x} v(t) d t, \\
\|v\|_{C_{1}}:=\|v\|_{E^{*}}, & \forall v \in C_{1} .
\end{array}
$$

Then, we have (see 21, Lemma 3])

$$
\|v\|_{V}^{2} \simeq\|v\|_{E}^{2}+\left\|\beta v^{\prime}\right\|_{\left(C_{0}, C_{1}\right)_{1 / 2,2}}^{2}
$$

where the norm $\|\cdot\|_{\left(C_{0}, C_{1}\right)_{1 / 2,2}}$ is defined by interpolation, similarly to (2.17). Actually, $\|\cdot\|_{\left(C_{0}, C_{1}\right)_{1 / 2,2}}$ plays the role of a $(-1 / 2)$-order norm and it is substantially independent of the coefficients $\varepsilon$ and $\rho$. Roughly speaking, one could think of $\|\cdot\|_{\left(C_{0}, C_{1}\right)_{1 / 2,2}}$ as $|\beta|^{-1 / 2}\|\cdot\|_{-1 / 2}$; this is stated properly in the next proposition.

Proposition 2.2. We have

$$
|\beta|^{1 / 2}|v|_{H^{1 / 2}} \preceq\left\|\beta v^{\prime}\right\|_{\left(C_{0}, C_{1}\right)_{1 / 2,2}} \preceq|\beta|^{1 / 2}\|v\|_{H_{00}^{1 / 2}}, \quad \forall v \in H_{0}^{1} .
$$

The proof of (2.23) can be found in [20] or [21, Theorem 2]. Together with (2.22), (2.23) motivates (1.3).

As a corollary of the previous results, $\|\cdot\|_{V}$ bounds $\|\cdot\|_{L^{2}}$, and the constant in the inequality depends on the coefficients $\varepsilon, \beta$ and $\rho$. Indeed, by definition, $\rho\|v\|_{L^{2}}^{2} \leq$ $\|v\|_{E}^{2} \leq\|v\|_{V}^{2}$; making use of the Poincaré inequality $\varepsilon L^{-2}\|v\|_{L^{2}}^{2} \preceq\|v\|_{E}^{2} \leq\|v\|_{V}^{2}$; we also have (2.20): $|\beta| L^{-1}\left(1+\log ^{+}(P e)\right)^{-1}\|v\|_{L^{2}}^{2} \preceq\|v\|_{V}^{2}$. Summarizing,

$$
\left(\varepsilon L^{-2}+|\beta| L^{-1}\left(1+\log ^{+}(P e)\right)^{-1}+\rho\right)\|v\|_{L^{2}}^{2} \preceq\|v\|_{V}^{2}, \quad \forall v \in H_{0}^{1} .
$$

Another corollary is that we can state meaningful a-priori bounds for the exact solution $u$ of (1.1). For example, (2.21), (2.20) and (2.23) yield

$$
\|u\|_{V} \preceq\left(1+\log ^{+}(P e)\right)^{1 / 2}|\beta|^{-1 / 2}\|f\|_{H^{-1 / 2}},
$$

where $\|\cdot\|_{H^{-1 / 2}}$ is the dual of $L^{-1 / 2}\|\cdot\|_{L^{2}}+|\cdot|_{H^{1 / 2}}$ (see [13, §12.1]). The estimate above expresses stability in the advection-dominated regime, uniformly with respect to $\rho \geq 0$ and with a weak $(\log (P e))^{1 / 2}$ growth for $P e \rightarrow+\infty$.

Finally, the proof of Lemma 2.1 is given below.

Proof of Lemma 2.1. We first give the proof of (2.19) for $T=T^{-}$. Let $\mu$ be a continuous weight function such that $\mu(0)=0, \mu^{\prime}=h_{T}^{-1}$ on $T$ and $\mu^{\prime}=0$ elsewhere. Therefore $\mu$ is piecewise linear and $0 \leq \mu \leq 1$ on $\Omega$. Given $v \in H_{0}^{1}$, integrating by parts and using the Cauchy-Schwartz inequality we have

$$
|\beta| h_{T}^{-1}\|v\|_{L^{2}(T)}^{2}=\int_{\Omega}|\beta| \mu^{\prime} v^{2}=-2 \int_{\Omega} \mu v|\beta| v^{\prime} \leq 2\|\mu v\|_{E}\left\|\beta v^{\prime}\right\|_{E^{*}}
$$

We have

$$
\|\mu v\|_{L^{2}} \leq\|v\|_{L^{2}}
$$

since $v$ is null at the endpoints of $\Omega$, from the Poincaré inequality we have $\|v\|_{L^{2}(T)} \preceq$ $h_{T}|v|_{H^{1}(T)}$, therefore

$$
|\mu v|_{H^{1}} \preceq\left(|v|_{H^{1}}+h_{T}^{-1}\|v\|_{L^{2}}\right) \preceq|v|_{H^{1}} .
$$


All of that gives

$$
\|\mu v\|_{E} \preceq\|v\|_{E} .
$$

Substituting (2.28) back in (2.25), we have

$$
|\beta| h_{T}^{-1}\|v\|_{L^{2}}^{2} \preceq\|v\|_{E}\left\|\beta v^{\prime}\right\|_{E^{*}}=\left\|\beta v^{\prime}\right\|_{C_{0}}\left\|\beta v^{\prime}\right\|_{C_{1}} .
$$

Using a classical result of the interpolation theory (see [14] or Lemma (a) in 22, $\S 1.10 .1])$ we can infer from (2.29) that

$$
|\beta|^{1 / 2} h_{T}^{-1 / 2}\|v\|_{L^{2}} \preceq\left\|\beta v^{\prime}\right\|_{\left(C_{0}, C_{1}\right)_{1 / 2,1}}, \quad \forall v \in H_{0}^{1} .
$$

Finally, using [21, Proposition 2] yields

$$
\left\|\beta v^{\prime}\right\|_{\left(C_{0}, C_{1}\right)_{1 / 2,1}} \preceq\left(1+\log ^{+}(P e)\right)^{1 / 2}\|v\|_{V}, \quad \forall v \in H_{0}^{1} .
$$

Clearly, (2.30) and (2.31) give (2.19).

For $T=T^{+}$the proof of (2.19) is similar. Analogously, taking $\mu(x)=x / L$ on $\Omega$, (2.20) is obtained.

2.3. Construction and properties of the needed interpolant. With the notation and definitions of $₫ 2.1$, we now introduce the usual nodal basis $\left\{\phi_{z}\right\}_{z \in \mathcal{Z}_{h}}$ for $V_{h}$, whence $\left\{\phi_{z}\right\}_{z \in \mathcal{Z}_{h}^{\circ}}$ is the nodal basis for $V_{h, 0}$. We also introduce the following interpolants: given $v \in H^{1}(\Omega)$ we define $\mathcal{I}_{h} v \in V_{h}$ as

$$
\mathcal{I}_{h} v:=\sum_{z \in \mathcal{Z}_{h}} \frac{\int_{\Omega} \phi_{z} v}{\int_{\Omega} \phi_{z}} \phi_{z}
$$

and $\mathcal{I}_{h, 0} v \in V_{h, 0}$ as

$$
\mathcal{I}_{h, 0} v:=\sum_{z \in \mathcal{Z}_{h}^{\circ}} \frac{\int_{\Omega} \phi_{z} v}{\int_{\Omega} \phi_{z}} \phi_{z} .
$$

Let $T \in \mathcal{T}_{h}$ and let $\omega_{T}$ be the union of $T$ and the (one or two) adjacent elements. From the definition (2.32) we easily get the local stability property

$$
\left\|\mathcal{I}_{h} v\right\|_{L^{2}(T)} \preceq\|v\|_{L^{2}\left(\omega_{T}\right)}, \forall v \in L^{2} .
$$

If $v_{\mid \omega_{T}}$ is constant, then $\left(\mathcal{I}_{h} v\right)_{\mid T}=v_{\mid \omega_{T}}$. Then, thanks to the Bramble-Hilbert lemma and the standard scaling argument, we have the local estimates

$$
\begin{aligned}
\left|\mathcal{I}_{h} v\right|_{H^{s}(T)} & \preceq|v|_{H^{s}\left(\omega_{T}\right)}, & & \forall v \in H^{s}, \text { for } s=1 / 2 \text { or } s=1, \\
\left\|v-\mathcal{I}_{h} v\right\|_{L^{2}(T)} & \preceq h_{T}^{s}|v|_{H^{s}\left(\omega_{T}\right)}, & & \forall v \in H^{s}, \text { for } s=1 / 2 \text { or } s=1 .
\end{aligned}
$$

From the inverse inequalities $h_{T}\left|\mathcal{I}_{h} v\right|_{H^{1}(T)} \preceq\left\|\mathcal{I}_{h} v\right\|_{L^{2}(T)}$ and $h_{T}^{1-s}\left|\mathcal{I}_{h} v\right|_{H^{1}(T)} \preceq$ $\left|\mathcal{I}_{h} v\right|_{H^{s}(T)}$, and from (2.34)-(2.35), we also get

$$
\begin{aligned}
h_{T}\left|\mathcal{I}_{h} v\right|_{H^{1}(T)} & \preceq\|v\|_{L^{2}\left(\omega_{T}\right)}, & & \forall v \in L^{2}, \\
h_{T}^{1-s}\left|\mathcal{I}_{h} v\right|_{H^{1}(T)} & \preceq|v|_{H^{s}\left(\omega_{T}\right)}, & & \forall v \in H^{s}, \text { for } s=1 / 2 \text { or } s=1 .
\end{aligned}
$$

Let $z \in \mathcal{Z}_{h}$ and let $T$ be an element to which $z$ belongs; using [23, Lemma 3.1], $\left(v-\mathcal{I}_{h} v\right)^{2}(z) \preceq h_{T}^{-1}\left\|v-\mathcal{I}_{h} v\right\|_{L^{2}(T)}^{2}+\left\|v-\mathcal{I}_{h} v\right\|_{L^{2}(T)}\left|v-\mathcal{I}_{h} v\right|_{H^{1}(T)}, \quad \forall v \in H^{1}$, and, using (2.35)-(2.36), we get

$$
\begin{aligned}
& \left(v-\mathcal{I}_{h} v\right)^{2}(z) \preceq\|v\|_{L^{2}\left(\omega_{T}\right)}|v|_{H^{1}\left(\omega_{T}\right)}, \\
& \left(v-\mathcal{I}_{h} v\right)^{2}(z) \preceq h_{T}|v|_{H^{1}\left(\omega_{T}\right)}^{2} .
\end{aligned}
$$


From the previous local estimates, we infer the global estimates that hold for all $v \in H^{1}$,

$$
\begin{aligned}
&\left\|\mathcal{I}_{h} v\right\|_{L^{2}(\Omega)} \preceq\|v\|_{L^{2}(\Omega)}, \\
& \sum_{T \in \mathcal{I}_{h}} h_{T}^{2}\left|\mathcal{I}_{h} v\right|_{H^{1}(T)}^{2} \preceq\|v\|_{L^{2}(\Omega)}^{2}, \\
& \sum_{T \in \mathcal{T}_{h}} h_{T}^{2-2 s}\left|\mathcal{I}_{h} v\right|_{H^{1}(T)}^{2} \preceq|v|_{H^{s}(\Omega)}^{2}, \quad \text { for } s=1 / 2 \text { or } s=1, \\
& \sum_{T \in \mathcal{T}_{h}} h_{T}^{-2 s}\left\|v-\mathcal{I}_{h} v\right\|_{L^{2}(T)}^{2} \preceq|v|_{H^{s}(\Omega)}^{2}, \quad \text { for } s=1 / 2 \text { or } s=1,
\end{aligned}
$$

and, for the nodal error,

$$
\begin{aligned}
\sum_{z \in \mathcal{Z}_{h}}\left(v-\mathcal{I}_{h} v\right)^{2}(z) & \preceq\|v\|_{L^{2}(\Omega)}|v|_{H^{1}(\Omega)}, \\
\sum_{z \in \mathcal{Z}_{h}} h_{z}^{-1}\left(v-\mathcal{I}_{h} v\right)^{2}(z) & \preceq|v|_{H^{1}(\Omega)}^{2},
\end{aligned}
$$

for all $v \in H^{1}$

Consider now $\mathcal{I}_{h, 0}$. Note that

$$
\mathcal{I}_{h} v-\mathcal{I}_{h, 0} v=\frac{\int_{\Omega} \phi_{0} v}{\int_{\Omega} \phi_{0}} \phi_{0}+\frac{\int_{\Omega} \phi_{L} v}{\int_{\Omega} \phi_{L}} \phi_{L}, \quad \forall v \in L^{2} .
$$

Then, we have for all $v$ and $T=T^{-}$or $T=T^{+}$,

$$
\left\|\mathcal{I}_{h} v-\mathcal{I}_{h, 0} v\right\|_{L^{2}(T)} \preceq\|v\|_{L^{2}(T)}, \quad \forall v \in L^{2},
$$

while $\mathcal{I}_{h} v$ and $\mathcal{I}_{h, 0} v$ coincide on $T \in \mathcal{T}_{h}^{\circ}$. For the inverse inequality $\left(h_{T} \mid \mathcal{I}_{h} v-\right.$ $\left.\left.\mathcal{I}_{h, 0} v\right|_{H^{1}(T)} \preceq\left\|\mathcal{I}_{h} v-\mathcal{I}_{h, 0} v\right\|_{L^{2}(T)}\right)$ we also have

$$
h_{T}\left|\mathcal{I}_{h} v-\mathcal{I}_{h, 0} v\right|_{H^{1}(T)} \preceq\|v\|_{L^{2}(T)}, \quad \forall v \in L^{2} .
$$

When $v \in H_{0}^{1}(\Omega)$, thanks to the Poincaré inequality on $T=T^{-}$or $T=T^{+}$, we have $\|v\|_{L^{2}(T)} \leq h_{T}|v|_{H^{1}(T)}$; using this in (2.45) -(2.46) gives

$$
\begin{aligned}
\left\|\mathcal{I}_{h} v-\mathcal{I}_{h, 0} v\right\|_{L^{2}(T)} & \preceq h_{T}|v|_{H^{1}(T)}, \quad \forall v \in H_{0}^{1}, \\
\left|\mathcal{I}_{h} v-\mathcal{I}_{h, 0} v\right|_{H^{1}(T)} & \preceq|v|_{H^{1}(T)}, \quad \forall v \in H_{0}^{1} .
\end{aligned}
$$

Using (2.40)-(2.43), (2.45)-(2.47) and the triangle inequality, for each $v \in H_{0}^{1}(\Omega)$ we get the global estimates

$$
\begin{aligned}
\left\|\mathcal{I}_{h, 0} v\right\|_{L^{2}(\Omega)} & \preceq\|v\|_{L^{2}(\Omega)}, \\
\sum_{T \in \mathcal{I}_{h}} h_{T}^{2}\left|\mathcal{I}_{h, 0} v\right|_{H^{1}(T)}^{2} & \preceq\|v\|_{L^{2}(\Omega)}, \\
\sum_{T \in \mathcal{T}_{h}} h_{T}\left|\mathcal{I}_{h, 0} v\right|_{H^{1}(T)}^{2} & \preceq|v|_{H^{1 / 2}(\Omega)}^{2}+h_{T^{-}}^{-1}\|v\|_{L^{2}\left(T^{-}\right)}^{2}+h_{T^{+}}^{-1}\|v\|_{L^{2}\left(T^{+}\right)}^{2}, \\
\left|\mathcal{I}_{h, 0} v\right|_{H^{1}(\Omega)}^{2} & \preceq|v|_{H^{1}(\Omega)}^{2}, \\
\sum_{T \in \mathcal{I}_{h}} h_{T}^{-1}\left\|v-\mathcal{I}_{h, 0} v\right\|_{L^{2}(T)}^{2} & \preceq|v|_{H^{1 / 2}(\Omega)}^{2}+h_{T^{-}}^{-1}\|v\|_{L^{2}\left(T^{-}\right)}^{2}+h_{T^{+}}^{-1}\|v\|_{L^{2}\left(T^{+}\right)}^{2}, \\
\sum_{T \in \mathcal{T}_{h}} h_{T}^{-2}\left\|v-\mathcal{I}_{h, 0} v\right\|_{L^{2}(T)}^{2} & \preceq|v|_{H^{1}(\Omega)}^{2} .
\end{aligned}
$$


Since $\mathcal{I}_{h} v$ and $\mathcal{I}_{h, 0} v$ coincide on the internal nodes, from (2.44) we immediately have, for all $v \in H^{1}(\Omega)$,

$$
\begin{aligned}
& \sum_{z \in \mathcal{Z}_{h}^{\circ}}\left(v-I_{h, 0} v\right)^{2}(z)\left.\preceq\left|v \|_{L^{2}(\Omega)}\right| v\right|_{H^{1}(\Omega)}, \\
& \sum_{z \in \mathcal{Z}_{h}^{\circ}} h_{z}^{-1}\left(v-I_{h, 0} v\right)^{2}(z) \preceq|v|_{H^{1}(\Omega)}^{2} .
\end{aligned}
$$

What we need in the next section are estimates for the interpolant $I_{h, 0} v$ in terms of $\|v\|_{V}$. They are stated in the following lemma.

Lemma 2.3. Assuming (2.3), for any $v \in H_{0}^{1}$ we have

$$
\begin{gathered}
\sum_{T \in \mathcal{T}_{h}} \tau_{T}^{2} \max \left\{\rho,|\beta|\left(1+\log ^{+}(P e)\right)^{-1} h_{T}^{-1}, \varepsilon h_{T}^{-2}\right\}\left\|\beta\left(I_{h, 0} v\right)^{\prime}\right\|_{L^{2}(T)}^{2} \preceq\|v\|_{V}^{2}, \\
\sum_{T \in \mathcal{T}_{h}} \max \left\{\rho,|\beta|\left(1+\log ^{+}(P e)\right)^{-1} h_{T}^{-1}, \varepsilon h_{T}^{-2}\right\}\left\|v-I_{h, 0} v\right\|_{L^{2}(T)}^{2} \preceq\|v\|_{V}^{2}, \\
\sum_{z \in \mathcal{Z}_{h}^{\circ}} \max \left\{\rho^{1 / 2} \varepsilon^{1 / 2},|\beta|\left(1+\log ^{+}(P e)\right)^{-1}, \varepsilon h_{z}^{-1}\right\}\left(v-I_{h, 0} v\right)^{2}(z) \preceq\|v\|_{V}^{2} .
\end{gathered}
$$

Proof. From (2.49) we get

$$
\rho \sum_{T \in \mathcal{T}_{h}} \tau_{T}^{2}\left\|\beta\left(I_{h, 0} v\right)^{\prime}\right\|_{L^{2}(T)}^{2} \preceq \rho\|v\|_{L^{2}(\Omega)}^{2},
$$

from (2.50), (2.19), (2.23) and (2.22), we get

$$
\begin{aligned}
\sum_{T \in \mathcal{T}_{h}} & \tau_{T}^{2}|\beta| h_{T}^{-1}\left\|\beta\left(I_{h, 0} v\right)^{\prime}\right\|_{L^{2}(T)}^{2} \\
& \preceq\left|\beta\left\|\left.v\right|_{H^{1 / 2}(\Omega)} ^{2}+|\beta| h_{T^{-}}^{-1}\right\| v\left\|_{L^{2}\left(T^{-}\right)}^{2}+|\beta| h_{T^{+}}^{-1}\right\| v \|_{L^{2}\left(T^{+}\right)}^{2}\right. \\
& \preceq\left(1+\log ^{+}(P e)\right)\|v\|_{V}^{2},
\end{aligned}
$$

and, from (2.51), we get

$$
\varepsilon \sum_{T \in \mathcal{T}_{h}} \tau_{T}^{2} h_{T}^{-2}\left\|\beta\left(I_{h, 0} v\right)^{\prime}\right\|_{L^{2}(T)}^{2} \preceq \varepsilon|v|_{H^{1}(\Omega)}^{2} .
$$

From (2.58)-(2.60) we get (2.55).

In a similar way, from (2.48), (2.52) with (2.19), (2.23), (2.22), and (2.53), we obtain (2.56).

From (2.54) we get

$$
\begin{aligned}
\rho^{1 / 2} \varepsilon^{1 / 2} \sum_{z \in \mathcal{Z}_{h}^{\circ}}\left(v-I_{h, 0} v\right)^{2}(z) & \preceq \rho^{1 / 2}\|v\|_{L^{2}(\Omega)} \cdot \varepsilon^{1 / 2}|v|_{H^{1}(\Omega)} \\
& \preceq \rho\|v\|_{L^{2}(\Omega)}^{2}+\varepsilon|v|_{H^{1}(\Omega)}^{2},
\end{aligned}
$$

similarly, still from (2.54), we have

$$
\varepsilon \sum_{z \in \mathcal{Z}_{h}^{\circ}} h_{z}^{-1}\left(v-I_{h, 0} v\right)^{2}(z) \preceq \varepsilon|v|_{H^{1}(\Omega)}^{2} .
$$

It remains to prove

$$
|\beta| \sum_{z \in \mathcal{Z}_{h}^{\circ}}\left(v-I_{h, 0} v\right)^{2}(z) \preceq\left(1+\log ^{+}(P e)\right)\|v\|_{V}^{2},
$$


and, after that, (2.57) follows from (2.61) - (2.63). The proof of (2.63) is the technical part. First, let $\bar{v}$ be the mean value of $v$. Since $I_{h, 0} \bar{v}=\bar{v}$ on the internal nodes, using (2.54) we get, for all $v \in H_{0}^{1}$,

$$
\sum_{z \in \mathcal{Z}_{h}^{\circ}}\left(v-I_{h, 0} v\right)^{2}(z) \preceq\|v-\bar{v}\|_{L^{2}(\Omega)}|v-\bar{v}|_{H^{1}(\Omega)} .
$$

Moreover,

$$
\begin{aligned}
\|v-\bar{v}\|_{L^{2}(\Omega)} & =\left\|v^{\prime}\right\|_{H^{-1}} \equiv \sup _{w \in H_{0}^{1}} \frac{\int_{\Omega} v^{\prime} w}{|w|_{H^{1}(\Omega)}} \\
|v-\bar{v}|_{H^{1}(\Omega)} & =\left\|v^{\prime}\right\|_{L^{2}(\Omega)}
\end{aligned}
$$

and therefore

$$
\sum_{z \in \mathcal{Z}_{h}^{\circ}}\left(v-I_{h, 0} v\right)^{2}(z) \preceq\left\|v^{\prime}\right\|_{H^{-1}}\left\|v^{\prime}\right\|_{L^{2}(\Omega)} .
$$

From (2.64) and thanks to a result of [14] (see also Lemma (a) in [22, §1.10.1]), we obtain

$$
\sum_{z \in \mathcal{Z}_{h}^{\circ}}\left(v-I_{h, 0} v\right)^{2}(z) \preceq\left\|v^{\prime}\right\|_{\left(H^{-1}, L^{2}\right)_{1 / 2,1}} .
$$

Moreover, reasoning as for the proof of Proposition 2.2 (or [21, Theorem 2]) we can obtain $|\beta|^{1 / 2}\left\|v^{\prime}\right\|_{\left(H^{-1}, L^{2}\right)_{1 / 2,1}} \leq\left\|\beta v^{\prime}\right\|_{\left(C_{0}, C_{1}\right)_{1 / 2,1}}$ (this is indeed a variation of the first inequality of (2.23) $)$. Recalling (2.31), from (2.65) we actually get (2.63), and this concludes the proof.

2.4. Construction of the a-posteriori estimators. This section is devoted to the proof of the a-posteriori estimates for (11). Our estimators (from below and from above) are defined as

$$
\begin{aligned}
& \check{\eta}_{V}:=\left(\sum_{T \in \mathcal{T}_{h}} \check{\eta}_{V, T}^{2}+\sum_{z \in \mathcal{Z}_{h}^{\circ}} \check{\eta}_{V, z}^{2}\right)^{1 / 2}, \\
& \hat{\eta}_{V}:=\left(\sum_{T \in \mathcal{T}_{h}} \hat{\eta}_{V, T}^{2}+\sum_{z \in \mathcal{Z}_{h}^{\circ}} \hat{\eta}_{V, z}^{2}\right)^{1 / 2},
\end{aligned}
$$

where

$$
\begin{aligned}
\check{\eta}_{V, T}^{2} & =\min \left\{\rho^{-1},|\beta|^{-1} h_{T}, \varepsilon^{-1} h_{T}^{2}\right\}\left\|f-\mathrm{E} u_{h}\right\|_{L^{2}(T)}^{2}, \\
\check{\eta}_{V, z}^{2} & =\min \left\{\rho^{-1 / 2} \varepsilon^{-1 / 2},|\beta|^{-1}, \varepsilon^{-1} h_{z}\right\}\left(\varepsilon\left[u_{h}^{\prime}\right](z)\right)^{2}, \\
\hat{\eta}_{V, T}^{2} & =\min \left\{\rho^{-1},|\beta|^{-1}\left(1+\log ^{+}(P e)\right) h_{T}, \varepsilon^{-1} h_{T}^{2}\right\}\left\|f-\mathrm{E} u_{h}\right\|_{L^{2}(T)}^{2}, \\
\hat{\eta}_{V, z}^{2} & =\min \left\{\rho^{-1 / 2} \varepsilon^{-1 / 2},|\beta|^{-1}\left(1+\log ^{+}(P e)\right), \varepsilon^{-1} h_{z}\right\}\left(\varepsilon\left[u_{h}^{\prime}\right](z)\right)^{2} .
\end{aligned}
$$

We also define the local counterpart of the spaces $V, C_{0}$ and $C_{1}$ of 2.2 . Given an open interval $\omega=\left(x_{1}, x_{2}\right) \subset \Omega$, we set $A_{0}=A_{1}=V:=H_{0}^{1}(\omega)$, and $C_{0}(\omega):=$ 
$\left\{v \in L^{2}(\omega) \mid \int_{\omega} v=0\right\}, C_{1}(\omega):=H^{-1}(\omega)$, endowed with the norms

$$
\begin{aligned}
\|v\|_{A_{0}(\omega)} & :=\|v\|_{E(\omega)}, \\
\|v\|_{A_{1}(\omega)} & :=\left(\|v\|_{E(\omega)}^{2}+\left\|\beta v^{\prime}\right\|_{E^{*}(\omega)}^{2}\right)^{1 / 2} \\
\|v\|_{V(\omega)} & :=\|v\|_{\left(A_{0}(\omega), A_{1}(\omega)\right)_{1 / 2,2}}, \\
\|\phi\|_{C_{0}(\omega)} & :=\left\|\beta^{-1} \Phi\right\|_{E(\omega)}, \quad \text { where } \Phi(x)=\int_{x_{1}}^{x} \phi(t) d t, \\
\|\phi\|_{C_{1}(\omega)} & :=\|\phi\|_{E^{*}(\omega)},
\end{aligned}
$$

where $\|\cdot\|_{E(\omega)}$ is the energy norm restricted to $H_{0}^{1}(\omega)$ and $\|\cdot\|_{E^{*}(\omega)}$ is the corresponding dual norm. The definition of $\|\cdot\|_{\left(A_{0}(\omega), A_{1}(\omega)\right)_{1 / 2,2}}$ is the analogous of (2.17). Thanks to [21, Lemma 3], we still have

$$
\|v\|_{V(\omega)}^{2} \simeq\|v\|_{E(\omega)}^{2}+\left\|\beta w^{\prime}\right\|_{\left(C_{0}(\omega), C_{1}(\omega)\right)_{1 / 2,2}}^{2} .
$$

Lemma 2.4. Let $\left\{\omega_{i}\right\}_{i \in I}$ be a family of disjoint open subsets of $\Omega$, and $\bigcup_{i \in I} \overline{\omega_{i}}=\bar{\Omega}$. Let $v$ be a function on $\Omega$ such that $v_{\mid \omega_{i}} \in H_{0}^{1}\left(\omega_{i}\right)$. Then we have

$$
\|v\|_{V}^{2} \leq \sum_{i \in I}\left\|v_{\mid \omega_{i}}\right\|_{V\left(\omega_{i}\right)}^{2}
$$

Proof. Given $v_{0}(t), v_{1}(t)$ such that $v_{0}(t)+v_{1}(t)=v$ and $v_{0}(t)_{\mid \omega_{i}}, v_{1}(t)_{\mid \omega_{i}} \in H_{0}^{1}\left(\omega_{i}\right)$, we have

$$
\begin{aligned}
\|v\|_{\left(A_{0}, A_{1}\right)_{1 / 2,2}}^{2} & \leq \int_{0}^{+\infty} t^{-1}\left\|v_{0}(t)\right\|_{A_{0}}^{2}+t\left\|v_{1}(t)\right\|_{A_{1}}^{2} \frac{d t}{t} \\
& =\int_{0}^{+\infty}\left(\sum_{i \in I} t^{-1}\left\|v_{0}(t)\right\|_{A_{0}\left(\omega_{i}\right)}^{2}+t\left\|v_{1}(t)\right\|_{A_{1}\left(\omega_{i}\right)}^{2}\right) \frac{d t}{t} \\
& =\sum_{i \in I}\left(\int_{0}^{+\infty} t^{-1}\left\|v_{0}(t)\right\|_{A_{0}\left(\omega_{i}\right)}^{2}+t\left\|v_{1}(t)\right\|_{A_{1}\left(\omega_{i}\right)}^{2} \frac{d t}{t}\right) .
\end{aligned}
$$

Taking the infimum on $v_{0}, v_{1}$ we obtain

$$
\|v\|_{\left(A_{0}, A_{1}\right)_{1 / 2,2}}^{2} \leq \sum_{i \in I}\left\|v_{\mid \omega_{i}}\right\|_{\left(A_{0}\left(\omega_{i}\right), A_{1}\left(\omega_{i}\right)\right)_{1 / 2,2}}^{2}
$$

and then (2.69).

The following lemma adapts a construction of [23] or [1] to our needs.

Lemma 2.5. Let $\tilde{k}$ be a positive integer and let $\tilde{v}$ be a continuous piecewise polynomial of degree $\tilde{k}$ on $\mathcal{T}_{h}$, such that $\tilde{v}_{T}:=\tilde{v}_{\mid T}$ is null at the endpoints of $T, \forall T \in \mathcal{T}_{h}$. Then

$$
\begin{aligned}
&\left\|\tilde{v}_{T}\right\|_{V(T)} \preceq \max \left\{\rho,|\beta| h_{T}^{-1}, \varepsilon h_{T}^{-2}\right\}^{1 / 2}\left\|\tilde{v}_{T}\right\|_{L^{2}(T)}, \\
&\|\tilde{v}\|_{V} \preceq\left(\sum_{T \in \mathcal{T}_{h}} \max \left\{\rho,|\beta| h_{T}^{-1}, \varepsilon h_{T}^{-2}\right\}\left\|\tilde{v}_{T}\right\|_{L^{2}(T)}^{2}\right)^{1 / 2} .
\end{aligned}
$$

Similarly, let $\bar{v}:=\sum_{z \in \mathcal{Z}_{h}^{\circ}} \bar{v}_{z}$, such that $\bar{v}_{z}$ is the hat function supported in

$$
S_{z}:=\left[z-1 / 2 \cdot \min \left\{h_{z}, \varepsilon^{1 / 2} \rho^{-1 / 2}\right\}, z+1 / 2 \cdot \min \left\{h_{z}, \varepsilon^{1 / 2} \rho^{-1 / 2}\right\}\right],
$$




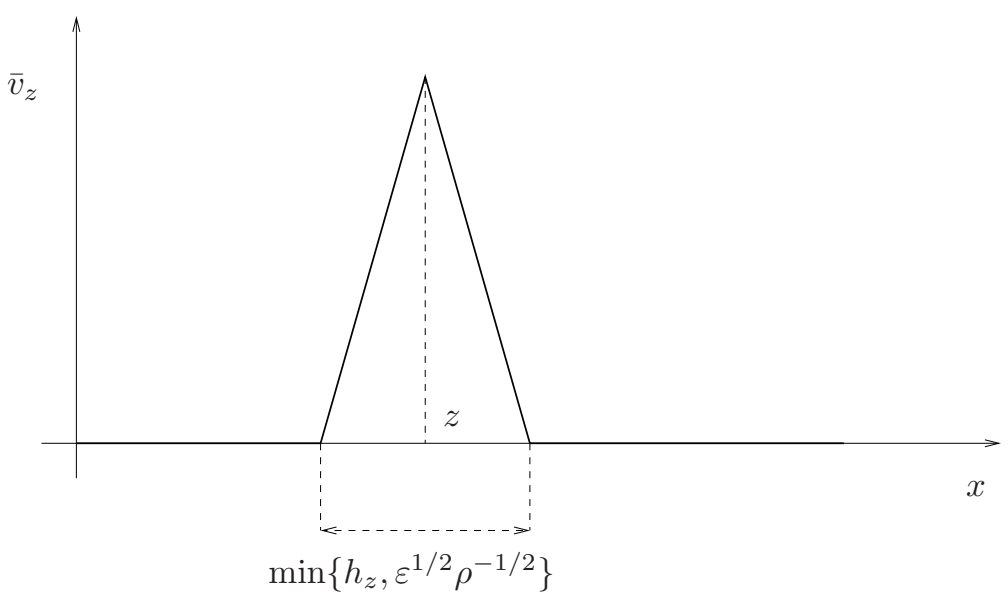

Figure 2. Construction of $\bar{v}_{z}$ in Lemma 2.5

as in Figure 2, Then

$$
\begin{aligned}
\max \left\{\rho,|\beta| h_{z}^{-1}, \varepsilon h_{z}^{-2}\right\}^{1 / 2}\|\bar{v}\|_{L^{2}\left(S_{z}\right)} & \preceq \max \left\{\rho^{1 / 2} \varepsilon^{1 / 2},|\beta|, \varepsilon h_{z}^{-1}\right\}^{1 / 2}|\bar{v}(z)|, \\
\sum_{T \in \mathcal{T}_{h}} \max \left\{\rho,|\beta| h_{T}^{-1}, \varepsilon h_{T}^{-2}\right\}\|\bar{v}\|_{L^{2}(T)}^{2} & \preceq \sum_{z \in \mathcal{Z}_{h}^{\circ}} \max \left\{\rho^{1 / 2} \varepsilon^{1 / 2},|\beta|, \varepsilon h_{z}^{-1}\right\}|\bar{v}(z)|^{2}, \\
\|\bar{v}\|_{V\left(S_{z}\right)} & \preceq \max \left\{\rho^{1 / 2} \varepsilon^{1 / 2},|\beta|, \varepsilon h_{z}^{-1}\right\}^{1 / 2}|\bar{v}(z)|, \\
\|\bar{v}\|_{V} & \preceq\left(\sum_{z \in \mathcal{Z}_{h}^{\circ}} \max \left\{\rho^{1 / 2} \varepsilon^{1 / 2},|\beta|, \varepsilon h_{z}^{-1}\right\}|\bar{v}(z)|^{2}\right)^{1 / 2} .
\end{aligned}
$$

Proof. We recall the usual inverse inequality

$$
\varepsilon^{1 / 2}\left|\tilde{v}_{T}\right|_{H^{1}(T)} \preceq \varepsilon^{1 / 2} h_{T}^{-1}\left\|\tilde{v}_{T}\right\|_{L^{2}(T)} .
$$

Then we get

$$
\begin{aligned}
\left\|\beta \tilde{v}_{T}^{\prime}\right\|_{C_{0}(T)} & =\left\|\tilde{v}_{T}\right\|_{E(T)} \preceq \max \left\{\rho, \varepsilon h_{T}^{-2}\right\}^{1 / 2}\left\|\tilde{v}_{T}\right\|_{L^{2}(T)}, \\
\left\|\beta \tilde{v}_{T}^{\prime}\right\|_{C_{1}(T)} & =\left\|\beta \tilde{v}_{T}^{\prime}\right\|_{E^{*}(T)} \preceq|\beta| h_{T}^{-1} \max \left\{\rho, \varepsilon h_{T}^{-2}\right\}^{-1 / 2}\left\|\tilde{v}_{T}\right\|_{L^{2}(T)},
\end{aligned}
$$

whence, using interpolation [22, Theorem 1.3.3.a],

$$
\left\|\beta \tilde{v}_{T}^{\prime}\right\|_{\left(C_{0}(T), C_{1}(T)\right)_{1 / 2,2}} \preceq|\beta|^{1 / 2} h_{T}^{-1 / 2}\left\|\tilde{v}_{T}\right\|_{L^{2}(T)} .
$$

Then, from (2.68), we obtain (2.70). Thanks to (2.69), we get (2.71).

For what concerns (2.72), it easily follows from the inverse inequality

$$
\left\|\bar{v}_{z}\right\|_{L^{2}\left(S_{z}\right)} \preceq\left|S_{z}\right|^{1 / 2}\left|\bar{v}_{z}(z)\right|,
$$

where $\left|S_{z}\right|:=\min \left\{h_{z}, \rho^{-1 / 2} \varepsilon^{1 / 2}\right\}$ is the length of the support $S_{z}$ of $\bar{v}_{z}$. Then, after squaring (2.72) and summing over the elements, since $h_{z} \simeq h_{T}$ when $z$ is a node of $T$, we get (2.73). 
By the same reasoning as for (2.70), we obtain

$$
\left\|\bar{v}_{z}\right\|_{V\left(S_{z}\right)} \preceq \max \left\{\rho,|\beta|\left|S_{z}\right|^{-1}, \varepsilon\left|S_{z}\right|^{-2}\right\}^{1 / 2}\left\|\bar{v}_{z}\right\|_{L^{2}\left(S_{z}\right)} .
$$

Then we can use (2.76) in (2.77), obtaining

$$
\left\|\bar{v}_{z}\right\|_{V\left(S_{z}\right)} \preceq \max \left\{\rho\left|S_{z}\right|,|\beta|, \varepsilon\left|S_{z}\right|^{-1}\right\}^{1 / 2}\left|\bar{v}_{z}(z)\right|,
$$

and then (2.74), since $\left|S_{z}\right|=\min \left\{h_{z}, \rho^{-1 / 2} \varepsilon^{1 / 2}\right\}$. Finally, thanks to (2.69), from (2.74) we get (2.75).

Our main result is stated in the next theorem.

Theorem 2.6. Let $u$ be the solution of (1.1) and let $u_{h}$ be given either by the plain Galerkin method (2.1) or by the SUPG method (2.2)-(2.3). Then

$$
\check{\eta}_{V} \preceq\left\|u-u_{h}\right\|_{V} \preceq \hat{\eta}_{V} .
$$

Proof. Thanks to (2.13), we have

$$
\left\|u-u_{h}\right\|_{V} \simeq\left\|f-\mathrm{E} u_{h}\right\|_{V^{*}}
$$

Then, we have to prove that

$$
\check{\eta}_{V} \preceq\left\|f-\mathrm{E} u_{h}\right\|_{V^{*}} \preceq \hat{\eta}_{V} .
$$

Let $I_{h, 0}$ be the interpolant operator defined in $\oiint 2.3$, Then

$$
\begin{aligned}
\sup _{v \in H_{0}^{1}} \frac{\left\langle f-\mathrm{Ł} u_{h}, v\right\rangle}{\|v\|_{V}} & \leq \sup _{v \in H_{0}^{1}} \frac{\left\langle f-\mathrm{E} u_{h}, I_{h, 0} v\right\rangle}{\|v\|_{V}}+\sup _{v \in H_{0}^{1}} \frac{\left\langle f-\mathrm{\complement} u_{h}, v-I_{h, 0} v\right\rangle}{\|v\|_{V}} \\
& =I+I I .
\end{aligned}
$$

The pairing $\left\langle f-\mathrm{E} u_{h}, I_{h, 0} v\right\rangle$, appearing in $I$, is either null, for the plain Galerkin method, or

$$
\left\langle f-\mathrm{E} u_{h}, I_{h, 0} v\right\rangle=\sum_{T \in \mathcal{T}_{h}} \tau_{T} \int_{T}\left(f-\mathrm{\iota} u_{h}\right)\left(\beta I_{h, 0} v\right)^{\prime}
$$

for the SUPG method. Thus the Cauchy-Schwartz inequality

$$
\int_{T}\left(f-\mathrm{E} u_{h}\right)\left(\beta I_{h, 0} v\right)^{\prime} \leq\left\|f-\mathrm{E} u_{h}\right\|_{L^{2}(T)}\left\|\left(\beta I_{h, 0} v\right)^{\prime}\right\|_{L^{2}(T)}
$$

and (2.55) yield

$$
I \preceq\left(\sum_{T \in \mathcal{T}_{h}} \eta_{V, T}^{2}\right)^{1 / 2}
$$

Integrating by parts, element by element, we get

$$
\begin{aligned}
\left\langle f-\mathrm{E} u_{h}, v-I_{h, 0} v\right\rangle= & \sum_{T \in \mathcal{T}_{h}} \int_{T}\left(f-\mathrm{E} u_{h}\right)\left(v-I_{h, 0} v\right) \\
& +\sum_{z \in \mathcal{Z}_{h}^{\circ}}-\varepsilon\left[u_{h}^{\prime}\right](z)\left(v-I_{h, 0} v\right)(z) .
\end{aligned}
$$

Then, by means of the Cauchy-Schwartz inequality and (2.56)-2.57) we now get

$$
I I \preceq\left(\sum_{T \in \mathcal{T}_{h}} \hat{\eta}_{V, T}^{2}+\sum_{z \in \mathcal{Z}_{h}^{\circ}} \hat{\eta}_{V, z}^{2}\right)^{1 / 2} .
$$

This gives the estimates from above stated in (2.79). 
Now, we turn our attention to the estimation below of $\left\|f-\mathrm{\iota} u_{h}\right\|_{V^{*}}$. By a usual scaling argument (recall that $f-\mathrm{\complement} u_{h}$ is a polynomial of degree at most $k$ in each element) we have

$$
\check{\eta}_{V, T}^{2} \simeq \int_{T} q_{T} \min \left\{\rho^{-1},|\beta|^{-1} h_{T}, \varepsilon^{-1} h_{T}^{-2}\right\}\left(f-\mathrm{E} u_{h}\right)^{2},
$$

where $q_{T}$ is the quadratic bubble on $T$ such that $\left\|q_{T}\right\|_{L^{\infty}(T)}=1$. Setting

$$
\tilde{v}:=\sum_{T \in \mathcal{T}_{h}} q_{T} \min \left\{\rho^{-1},|\beta|^{-1} h_{T}, \varepsilon^{-1} h_{T}^{-2}\right\}\left(f-\mathrm{L} u_{h}\right),
$$

we then have

$$
\sum_{T \in \mathcal{T}_{h}} \check{\eta}_{V, T}^{2} \simeq\left\langle f-\mathrm{E} u_{h}, \tilde{v}\right\rangle
$$

Moreover, since the function $\tilde{v}$ defined above is a piecewise polynomial of degree $\tilde{k}=k+2$, null at the nodes, we can apply Lemma 2.5, and in particular (2.71), obtaining

$$
\|\tilde{v}\|_{V} \preceq\left(\sum_{T \in \mathcal{T}_{h}} \check{\eta}_{V, T}^{2}\right)^{1 / 2}
$$

Therefore

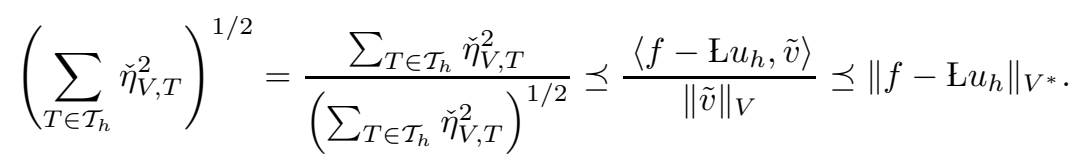

Similarly, we define $\bar{v}=\sum_{z \in \mathcal{Z}_{h}^{\circ}} \bar{v}_{z}$ as in Lemma 2.5, with

$$
\bar{v}_{z}(z):=\min \left\{\rho^{-1 / 2} \varepsilon^{-1 / 2},|\beta|^{-1}, \varepsilon^{-1} h_{x}^{-1}\right\} \varepsilon\left[u_{h}^{\prime}\right](z) .
$$

Then we have, integrating by parts,

$$
\begin{aligned}
\sum_{z \in \mathcal{Z}_{h}^{\circ}} \check{\eta}_{V, z}^{2} & =\sum_{z \in \mathcal{Z}_{h}^{\circ}} \varepsilon\left[u_{h}^{\prime}\right](z) \bar{v}_{z}(z) \\
& =\left\langle f-\mathrm{\complement} u_{h}, \bar{v}\right\rangle-\sum_{T \in \mathcal{T}_{h}} \int_{T}\left(f-\mathrm{L} u_{h}\right) \bar{v} \\
& =I+I I .
\end{aligned}
$$

Using (2.75) we get

$$
I \leq\left\|f-\mathrm{\complement} u_{h}\right\|_{V^{*}}\|\bar{v}\|_{V} \preceq\left\|f-\mathrm{\textrm {L }} u_{h}\right\|_{V^{*}}\left(\sum_{z \in \mathcal{Z}_{h}^{\circ}} \check{\eta}_{V, z}^{2}\right)^{1 / 2}
$$


and, for (2.73) and (2.85),

$$
\begin{aligned}
I I \leq & \left(\sum_{T \in \mathcal{T}_{h}} \min \left\{\rho^{-1},|\beta|^{-1} h_{T}, \varepsilon^{-1} h_{T}^{2}\right\}\left\|f-\mathrm{\iota} u_{h}\right\|_{L^{2}(T)}^{2}\right)^{1 / 2} \\
& \cdot\left(\sum_{T \in \mathcal{T}_{h}} \max \left\{\rho,|\beta| h_{T}^{-1}, \varepsilon h_{T}^{-2}\right\}\|\bar{v}\|_{L^{2}(T)}^{2}\right)^{1 / 2} \\
& \leq\left(\sum_{T \in \mathcal{T}_{h}} \check{\eta}_{V, T}^{2}\right)^{1 / 2}\left(\sum_{z \in \mathcal{Z}_{h}^{\circ}} \max \left\{\rho^{1 / 2} \varepsilon^{1 / 2},|\beta|, \varepsilon h_{x}^{-1}\right\}|\bar{v}(z)|^{2}\right)^{1 / 2} \\
& \left\|f-\mathrm{L} u_{h}\right\|_{V^{*}}\left(\sum_{T \in \mathcal{T}_{h}} \check{\eta}_{V, z}^{2}\right)^{1 / 2} .
\end{aligned}
$$

Using the last estimates in (2.86) we end up with

$$
\left(\sum_{T \in \mathcal{T}_{h}} \check{\eta}_{V, z}^{2}\right)^{1 / 2} \preceq\left\|f-\mathrm{\llcorner} u_{h}\right\|_{V^{*}} .
$$

Collecting (2.82) $-(2.87)$ and recalling (2.81) , we finally get (2.79).

2.5. One-dimensional numerical tests. In this section we test the a-posteriori error estimators (2.66)-(2.67), and compare them with those of [23].

We only perform tests in the advection-dominated regime (in the other regimes, when diffusion or the reaction-dominates, the present estimators coincide with those of 23). We consider here two simple model problems: a one-dimensional advectiondiffusion-reaction problem, with $\varepsilon=10^{-6}, \beta=\rho=1$,

$$
\left\{\begin{aligned}
-10^{-6} u^{\prime \prime}+u^{\prime}+u & =f \quad \text { in }(0,1), \\
u(0)=u(1) & =0,
\end{aligned}\right.
$$

and a one-dimensional advection-diffusion problem, with $\varepsilon=10^{-6}, \beta=1$ and $\rho=0$,

$$
\left\{\begin{aligned}
-10^{-6} u^{\prime \prime}+u^{\prime} & =f \quad \text { in }(0,1), \\
u(0)=u(1) & =0
\end{aligned}\right.
$$

for different right-hand sides $f$.

The theory of 23] encompasses (ADR but not (AD). Indeed, in 23] the problem is assumed to be $L^{2}$-coercive, uniformly with respect to the coefficients (in our onedimensional example, this means that the reaction coefficient $\rho$ is taken fixed and positive) and the estimates do not depend explicitly on $\rho$, but only on $\varepsilon$ and $\beta$. However, a straightforward generalization of 23] allows for any $\rho \geq 0$, and leads to the following (one-dimensional) estimators whose expression depends on all of the 
coefficients:

$$
\begin{aligned}
& \check{\eta}_{E}:=\left(\sum_{T \in \mathcal{T}_{h}} \check{\eta}_{E, T}^{2}+\sum_{z \in \mathcal{Z}_{h}^{\circ}} \check{\eta}_{E, z}^{2}\right)^{1 / 2}, \\
& \hat{\eta}_{E}:=\left(\sum_{T \in \mathcal{T}_{h}} \hat{\eta}_{E, T}^{2}+\sum_{z \in \mathcal{Z}_{h}^{\circ}} \hat{\eta}_{E, z}^{2}\right)^{1 / 2},
\end{aligned}
$$

where

$$
\begin{aligned}
\check{\eta}_{E, T}^{2} & =\frac{\min \left\{\rho^{-1}, \varepsilon^{-1} h_{T}^{2}\right\}}{1+\min \left\{|\beta|^{2} h_{T}^{2} \varepsilon^{-2},|\beta|^{2} \varepsilon^{-1} \rho^{-1}\right\}}\left\|f-\mathrm{L} u_{h}\right\|_{L^{2}(T)}^{2}, \\
\check{\eta}_{E, z}^{2} & =\frac{\min \left\{\rho^{-1 / 2} \varepsilon^{-1 / 2}, \varepsilon^{-1} h_{z},\right\}}{1+\min \left\{|\beta|^{2} h_{z}^{2} \varepsilon^{-2},|\beta|^{2} \varepsilon^{-1} \rho^{-1}\right\}}\left(\varepsilon\left[u_{h}^{\prime}\right](z)\right)^{2}, \\
\hat{\eta}_{E, T}^{2} & =\min \left\{\rho^{-1}, \varepsilon^{-1} h_{T}^{2}\right\}\left\|f-\mathrm{E} u_{h}\right\|_{L^{2}(T)}^{2}, \\
\hat{\eta}_{E, z}^{2} & =\min \left\{\rho^{-1 / 2} \varepsilon^{-1 / 2}, \varepsilon^{-1} h_{z}\right\}\left(\varepsilon\left[u_{h}^{\prime}\right](z)\right)^{2} .
\end{aligned}
$$

If $u_{h}$ is a plain Galerkin or SUPG discrete solution of the problem (1.1), one ha: 2 (as in [23, Proposition 4.1])

$$
\check{\eta}_{E} \preceq\left\|u-u_{h}\right\|_{E} \preceq \hat{\eta}_{E} .
$$

In what follows, $u_{h}$ is computed by a piecewise linear SUPG-FEM scheme (2.2), with stabilizing parameter

$$
\tau_{T}:=h_{T} /\left(2|\beta|_{T}\right)=h_{T} / 2 .
$$

The exact solution $u$ is also approximated numerically, on a fine mesh which fully resolves the smallest scales of the problem.

Our aim is to measure the effectivity indeces (e.i.) for estimators (2.88)-(2.89) and (2.66) -2.67), that is, the quantities

$$
\frac{\check{\eta}_{E}}{\left\|u-u_{h}\right\|_{E}} \quad \text { and } \quad \frac{\hat{\eta}_{E}}{\left\|u-u_{h}\right\|_{E}}
$$

and

$$
\frac{\check{\eta}_{V}}{\left\|u-u_{h}\right\|_{V}} \quad \text { and } \quad \frac{\hat{\eta}_{V}}{\left\|u-u_{h}\right\|_{V}}
$$

respectively.

\footnotetext{
${ }^{2} \mathrm{~A}$ proof of 2.90 can be given also imitating the one of Theorem 2.6 We sketch it in what follows, assuming a uniform mesh and considering the plain Galerkin method for the sake of simplicity. First, one has

$$
\left\|u-u_{h}\right\|_{E} \leq\left\|f-\mathrm{L} u_{h}\right\|_{E^{*}} \preceq\left(1+\min \left\{|\beta| h \varepsilon^{-1},|\beta| \varepsilon^{-1 / 2} \rho^{-1 / 2}\right\}\right)\left\|u-u_{h}\right\|_{E} ;
$$

indeed, the left bound above comes from the coercivity of $\mathrm{E}$, while the right bound follows from the Cauchy-Schwartz inequality and the Galerkin orthogonality. The second step is

$$
\hat{\eta}_{E} \preceq\left\|f-\mathrm{L} u_{h}\right\|_{E^{*}} \preceq \hat{\eta}_{E},
$$

which can be proved similarly to (2.81), replacing $\beta$ with 0 whenever $\beta$ appears in $\|\cdot\|_{V^{*}},\|\cdot\|_{V}$ and in the estimators. Together, the two estimates give (2.90).
} 


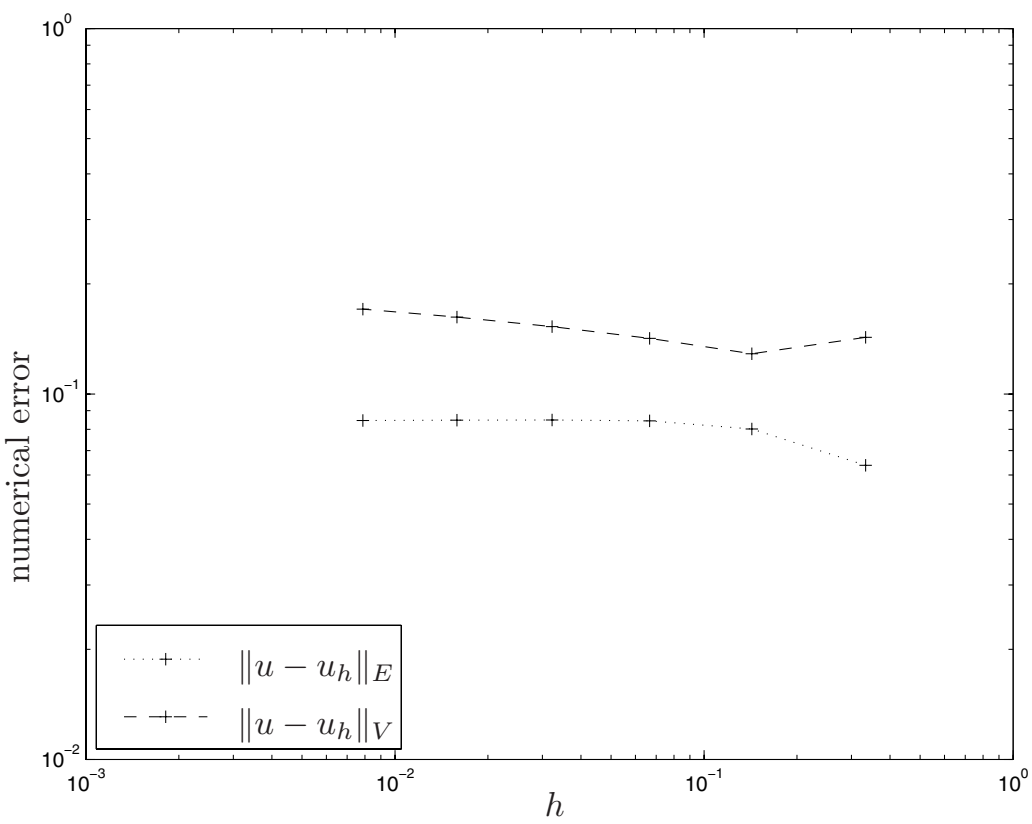

Figure 3. Numerical errors for problem (ADR), and $f(x)=$ $\cos (5 / 2 \pi x)$, on uniform meshes

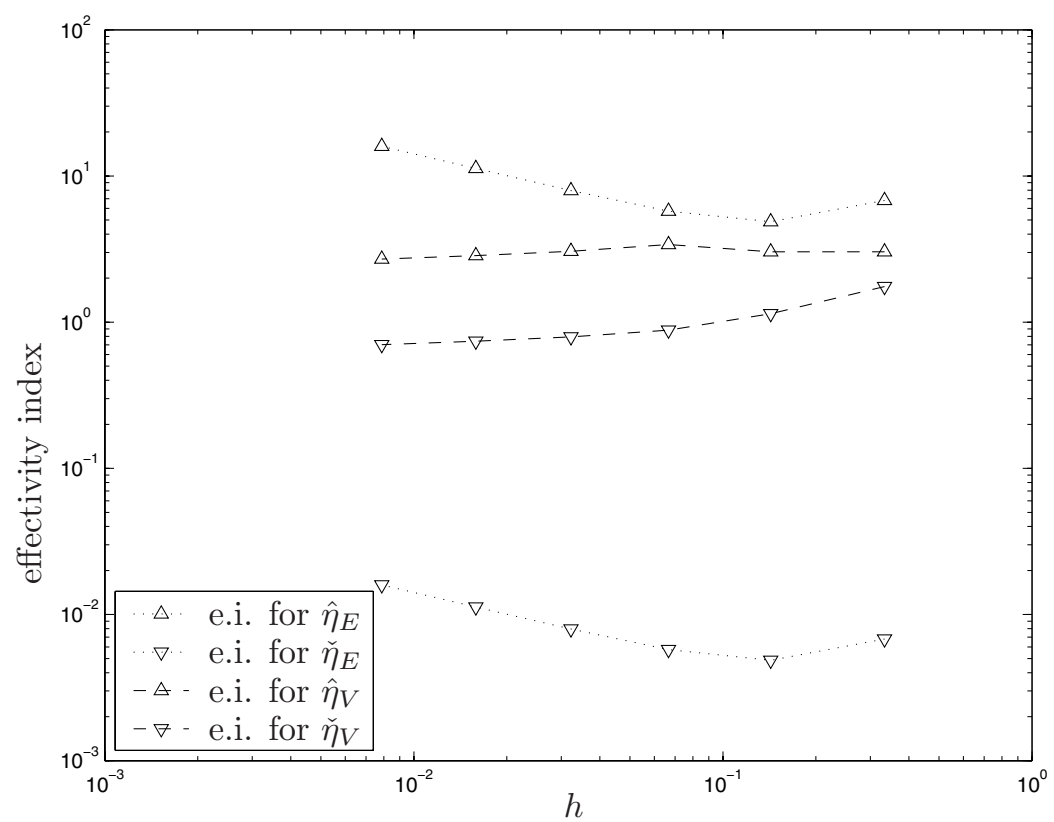

Figure 4. Effectivity indeces for the problem considered in Figure 3 


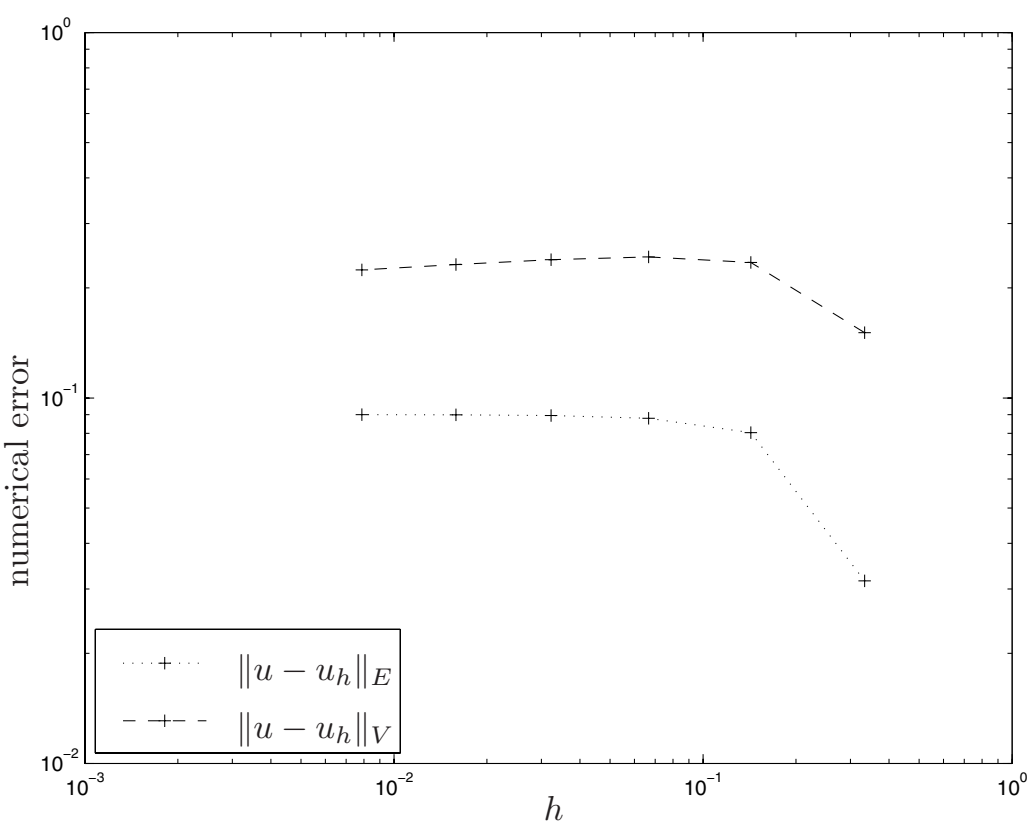

Figure 5. Numerical errors for problem (AD), and $f(x)=$ $\cos (5 / 2 \pi x)$, on uniform meshes

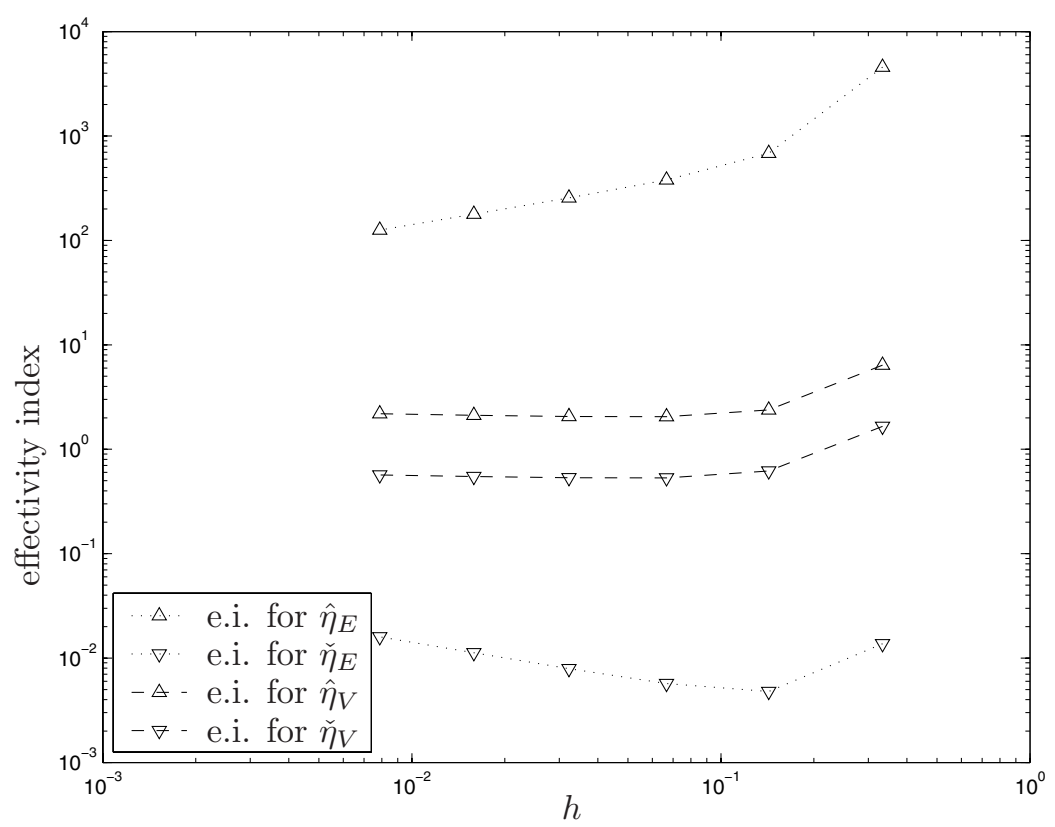

Figure 6. Effectivity indeces for the problem considered in Figure 5 
For the evaluation of $\|\cdot\|_{V}$ we adopt the following procedure: on a fine mesh we assemble the scalar product $\mathbf{A}_{0}$ and $\mathbf{A}_{1}$, corresponding to the norms in (2.15); then we compute a generalized eigenvalues decomposition

$$
\mathbf{A}_{1} * \mathbf{W}=\mathbf{A}_{0} * \mathbf{W} * \mathbf{D},
$$

where $\mathbf{W}$ and $\mathbf{D}$ denote the eigenvectors and the diagonal eigenvalue's matrices, respectively. Finally, we approximate the scalar product associated to $\|\cdot\|_{V}$ by the matrix $\mathbf{V}:=\left(\mathbf{A}_{0} * \mathbf{W}\right)^{t} * \mathbf{D}^{1 / 2} *\left(\mathbf{A}_{0} * \mathbf{W}\right)$. This procedure is motivated by the construction of interpolated norms as described in [13, §2.1].

In the first two tests we set $f(x)=\cos (5 / 2 \pi x)$, and we compute $u_{h}$ on a sequence of uniform meshes of $2^{i}$ nodes, with $i=2, \ldots, 7$. The numerical errors $\left\|u-u_{h}\right\|_{E}$ and $\left\|u-u_{h}\right\|_{V}$ are plotted in Figures 3 and 5, for (ADR) and (AD), respectively. The exact solution has a boundary layer at $x=1$, which is under-resolved on all of the meshes. For that reason, in Figures 3 and 5 we do not see any order convergence of the numerical solution $u_{h}$ towards the exact solution $u$. In Figures 4 and 6 we plot the e.i. for these two tests. We can see that $\hat{\eta}_{V}$ and $\check{\eta}_{V}$ give quite satisfactory results, with e.i. that are less than one order of magnitude close to the optimality (e.i.=1), and perform better than $\hat{\eta}_{E}$ and $\check{\eta}_{E}$. Notice the poor performance of $\hat{\eta}_{E}$ and $\check{\eta}_{E}$ for (AD) (recall that these estimators are based on the coercivity of the differential operator, which is a very weak condition when $\rho=0$ ).

In the second set of tests the exact solution $u$ has no layers, with $f(x)=x^{2} / 2+$ $x / 2-1 / 2$ for (ADR) and $f(x)=x-1 / 2$ for (AD). The meshes, as before, are uniform with $2^{i}$ nodes, $i=2, \ldots, 7$. Now we see the expected order of convergence for $\left\|u-u_{h}\right\|_{E}$ and $\left\|u-u_{h}\right\|_{V}$, which is 2 and 3/2 for (ADR) (see Figure 7) and 1 and $3 / 2$ for (AD) (see Figure 9), respectively. Now $\hat{\eta}_{E}$ is far from optimality, while the other estimators give good effectivity indeces (see Figures 8 and 10).

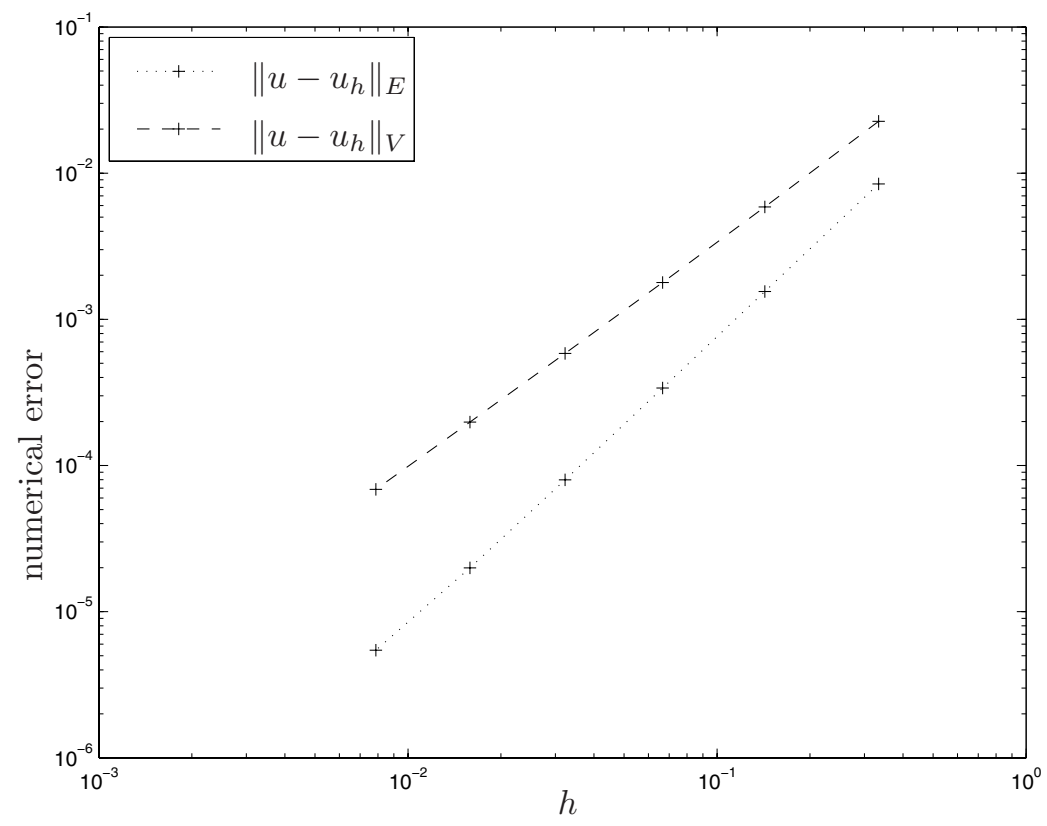

FiguRE 7. Numerical errors for problem ADR, and $f(x)=$ $x^{2} / 2+x / 2-1 / 2$, on uniform meshes 


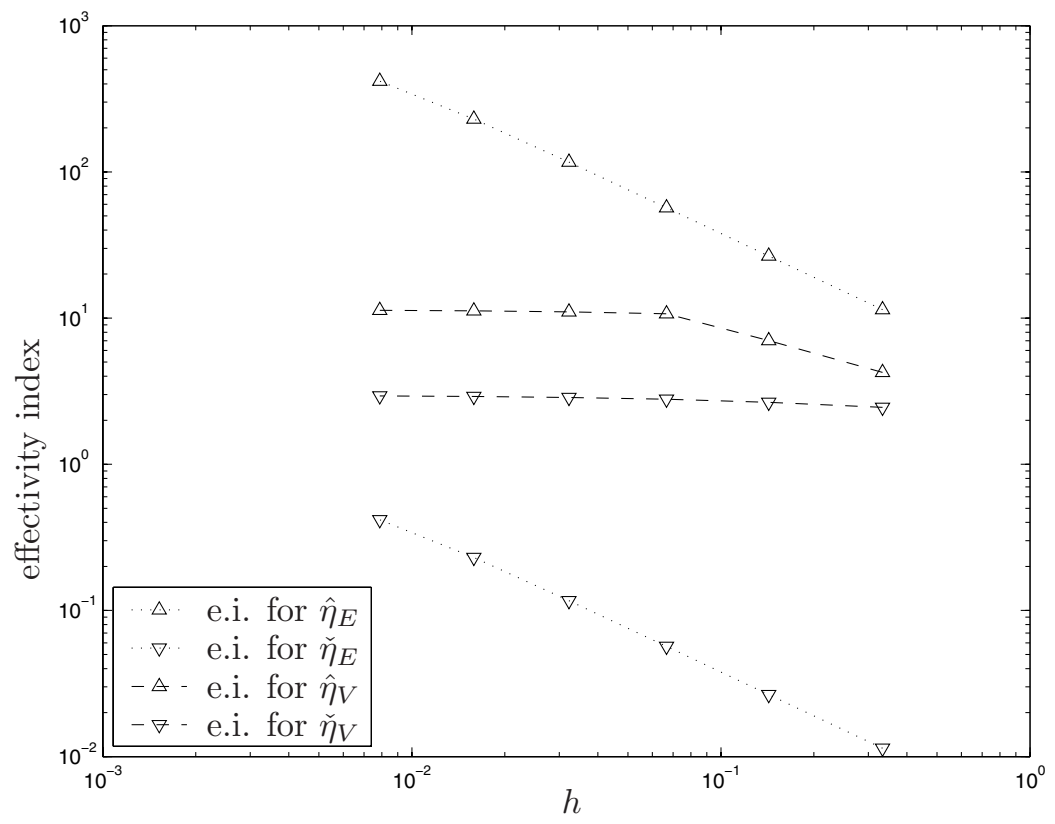

FiguRE 8. Effectivity indeces for the problem considered in Figure 7

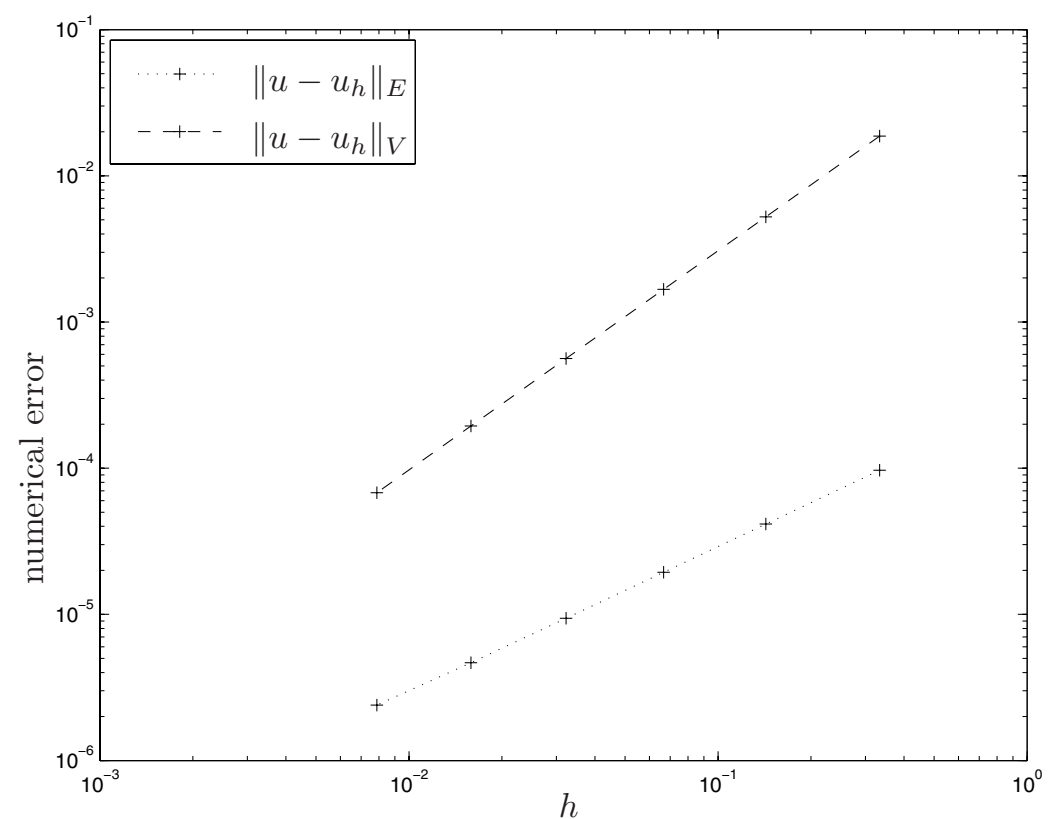

Figure 9. Numerical errors for problem (AD), and $f(x)=x-1 / 2$, on uniform meshes 


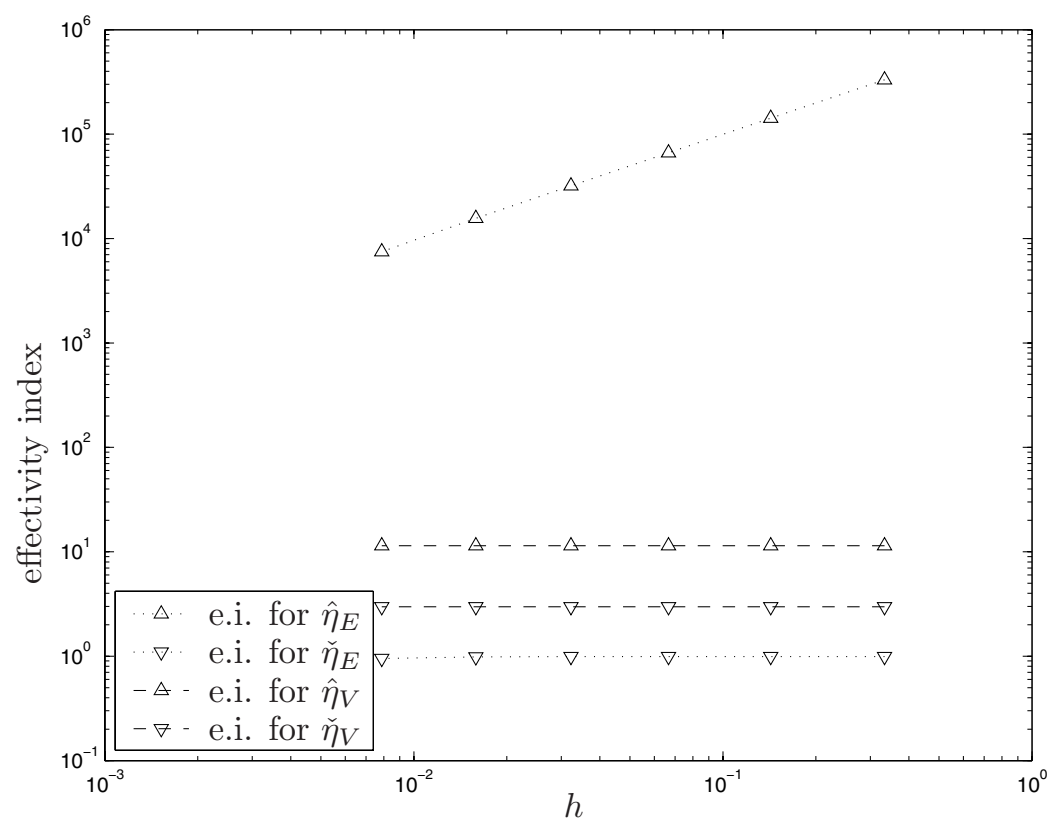

FiguRE 10. Effectivity indeces for the problem considered in Figure 9

In the last tests we consider an adaptive procedure, for both (ADR) and (AD), and $f(x)=\cos (5 / 2 \pi x)$. We follow the strategy of [23]: we start with a uniform mesh (5 elements) and, at each step, we compute the numerical solution $u_{h}$, we compute a local estimator, and mark for refinement the elements where the local estimator is larger than one half of the maximum of the local estimators. For an internal element $T=\left(z^{-}, z^{+}\right)$, the local estimators are taken as

$$
\hat{\eta}_{E, T}^{2}+\hat{\eta}_{E, z^{-}}^{2}+\hat{\eta}_{E, z^{+}}^{2}
$$

and

$$
\hat{\eta}_{V, T}^{2}+\hat{\eta}_{V, z^{-}}^{2}+\hat{\eta}_{V, z^{+}}^{2},
$$

respectively, with the obvious modification for the first and last element. The error histories are shown in Figure 11 for (ADR), and in Figure 13 for (AD). In both cases, the mesh is initially refined only in the layer region. When the boundary layer (at $x=1$ ) is captured, the refinement occurs in the whole domain, and the errors start decreasing. In Figures 12 and 14 we plot the e.i. for the two cases, and confirm that the estimators $\hat{\eta}_{E}$ and $\check{\eta}_{E}$ are not robust, at least when the layer is not resolved. 


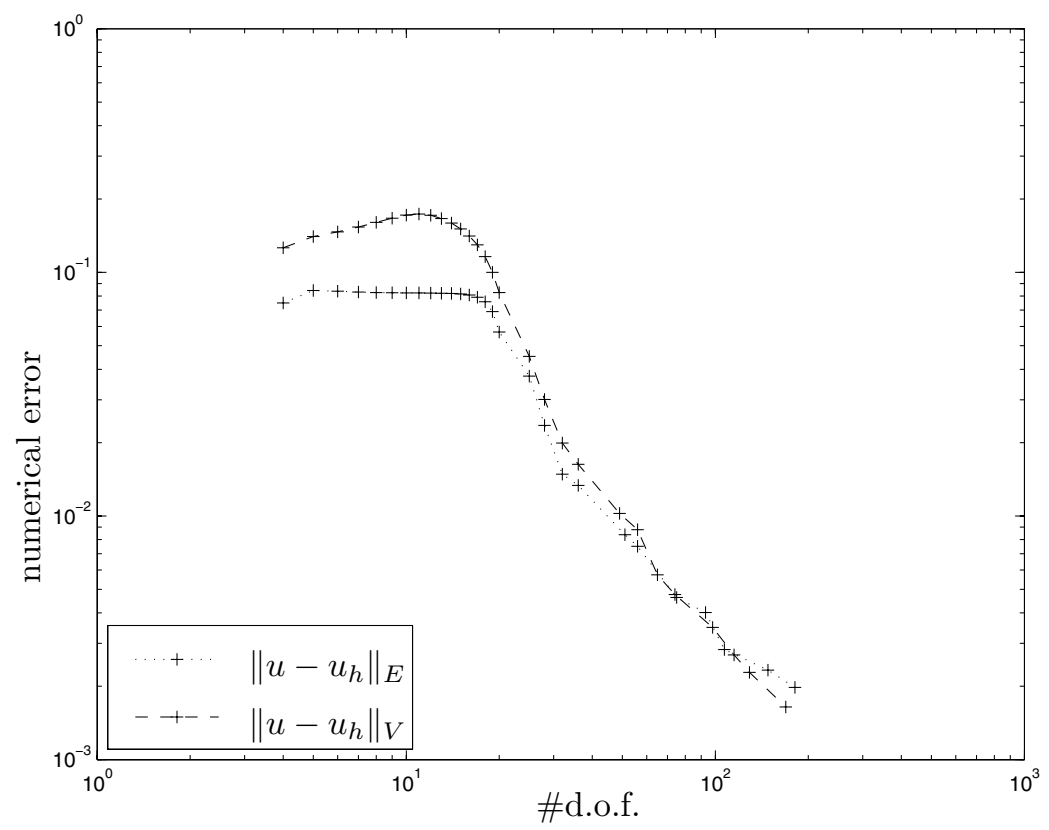

FiguRE 11. Numerical errors for problem (ADR), and $f(x)=$ $\cos (5 / 2 \pi x)$, with adaptive refinement

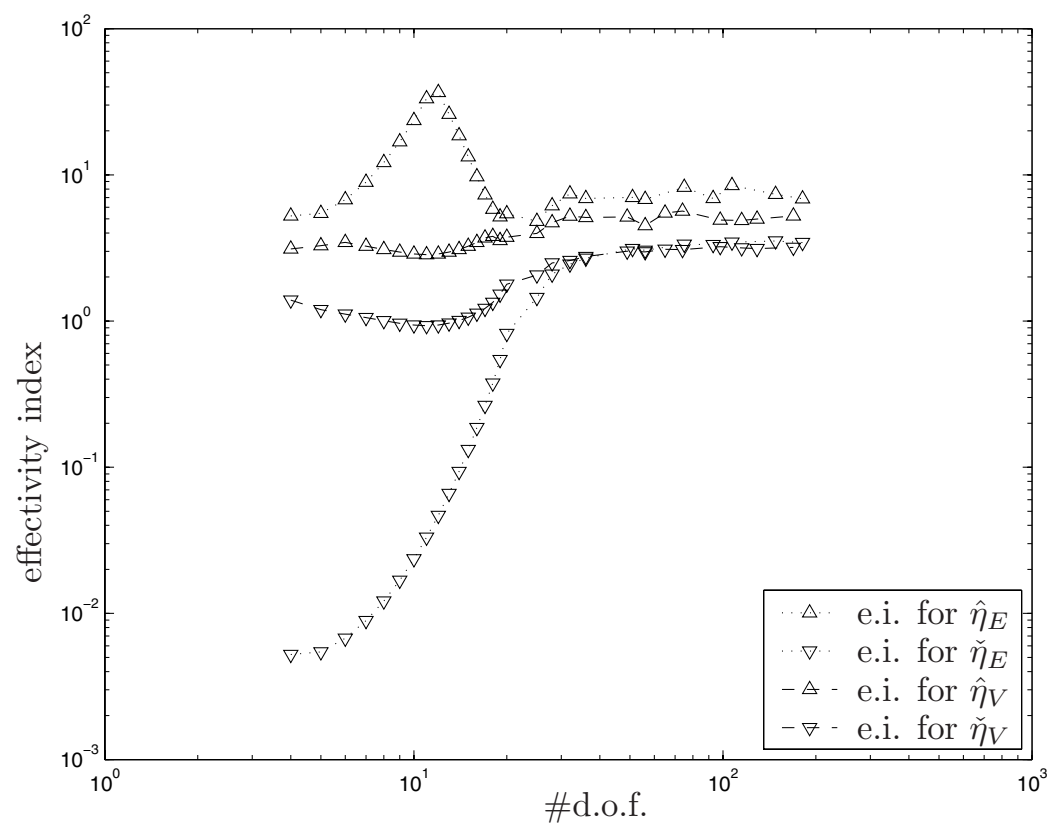

FIGURE 12. Effectivity indeces for the problem considered in Figure 11 


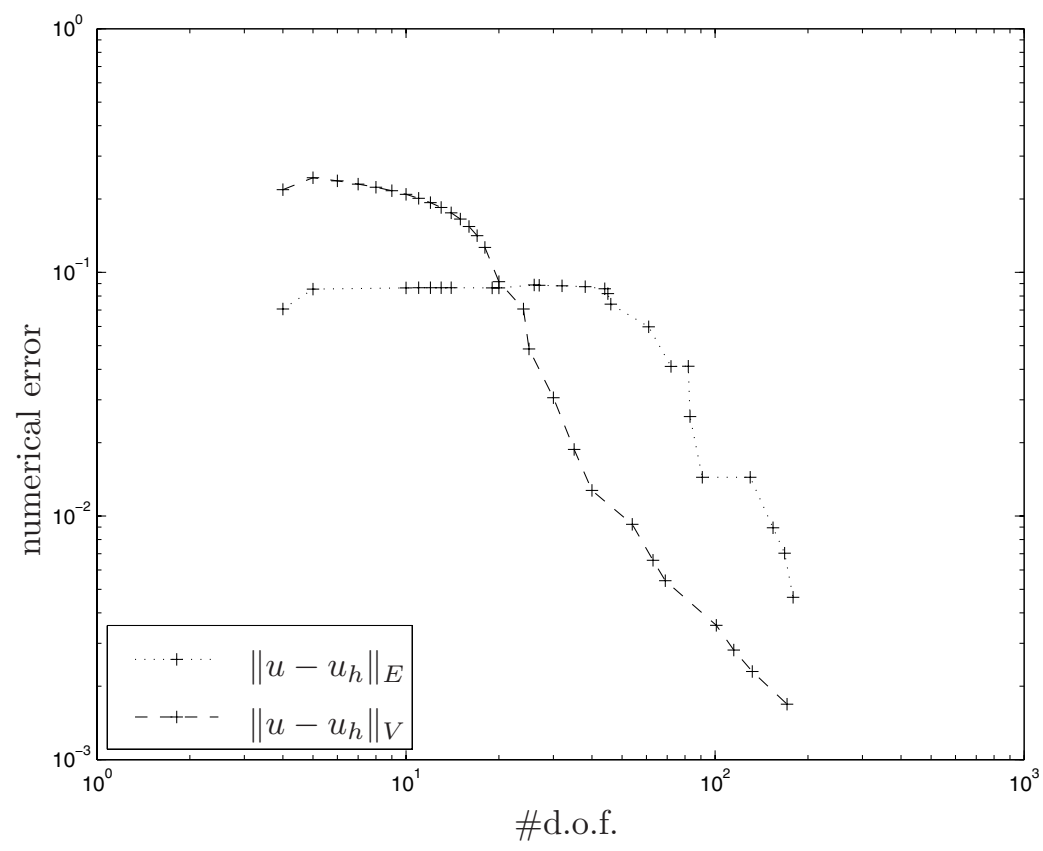

Figure 13. Numerical errors for problem (AD), and $f(x)=$ $\cos (5 / 2 \pi x)$, with adaptive refinement

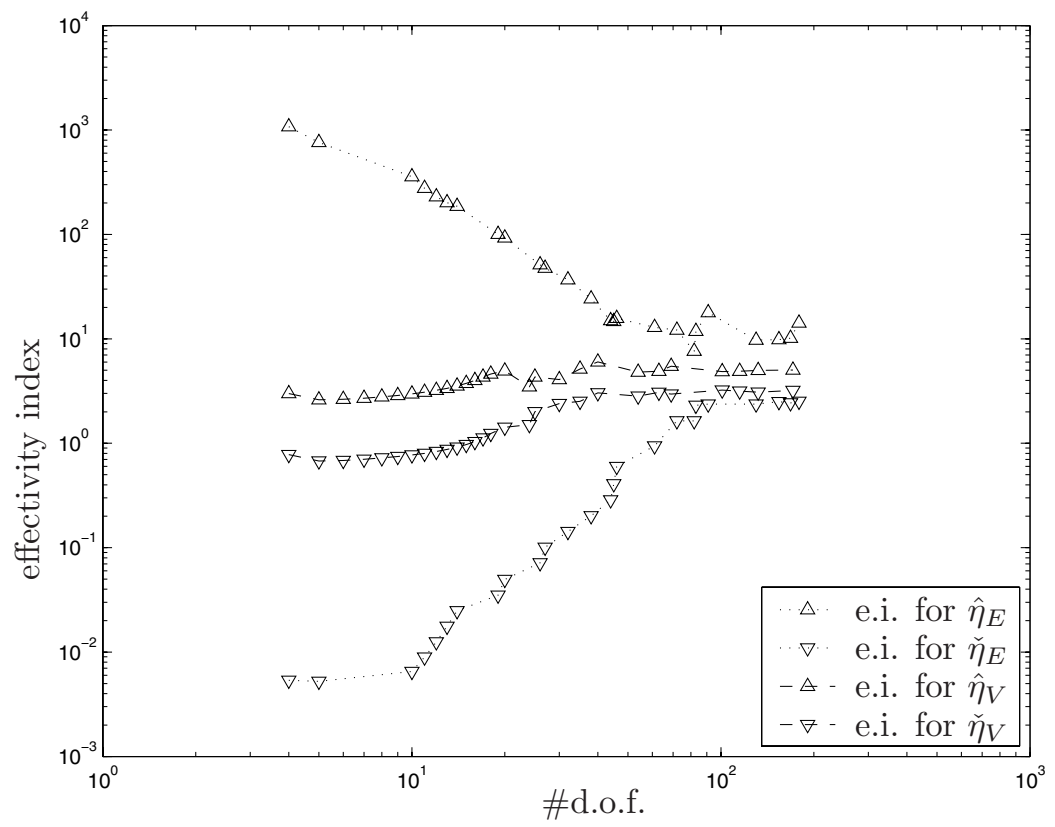

Figure 14. Effectivity indeces for the problem considered in Figure 13 
Finally, observe that, from $\|\cdot\|_{A_{i}} \geq\|\cdot\|_{E}$ and the definition of $\|\cdot\|_{V}$, one has $\|\cdot\|_{V} \geq\|\cdot\|_{E}$. Therefore

$$
\left\|u-u_{h}\right\|_{E} \preceq \hat{\eta}_{V} .
$$

Though trivial, (2.95) is interesting because, comparing (2.67) with (2.89), we also have $\hat{\eta}_{E} \geq \hat{\eta}_{V}$. Then, (2.95) is a more effective estimate from above of the error $\left\|u-u_{h}\right\|_{E}$ than the estimate $\left\|u-u_{h}\right\|_{E} \preceq \hat{\eta}_{E}$. That is, $\hat{\eta}_{V}$ is a better estimator of $\left\|u-u_{h}\right\|_{E}$ than $\hat{\eta}_{E}$. This is confirmed by the tests. For example, in Figure 15] we consider (AD) with $f(x)=\cos (5 / 2 \pi x)$ and compare the errors $\left\|u-u_{h}\right\|_{E}$ for the two adaptive procedures driven by the local estimators (2.93) and (2.94), respectively; the latter procedure gives meshes on which the error $\left\|u-u_{h}\right\|_{E}$ is in fact smaller. Improvements are seen also for (ADR (not shown).

A similar qualitative behavior of the estimators is observed when a different stabilizing parameter $\tau_{T}$ is chosen for SUPG, or when the plain Galerkin method is used for computing $u_{h}$ (the results are not shown).

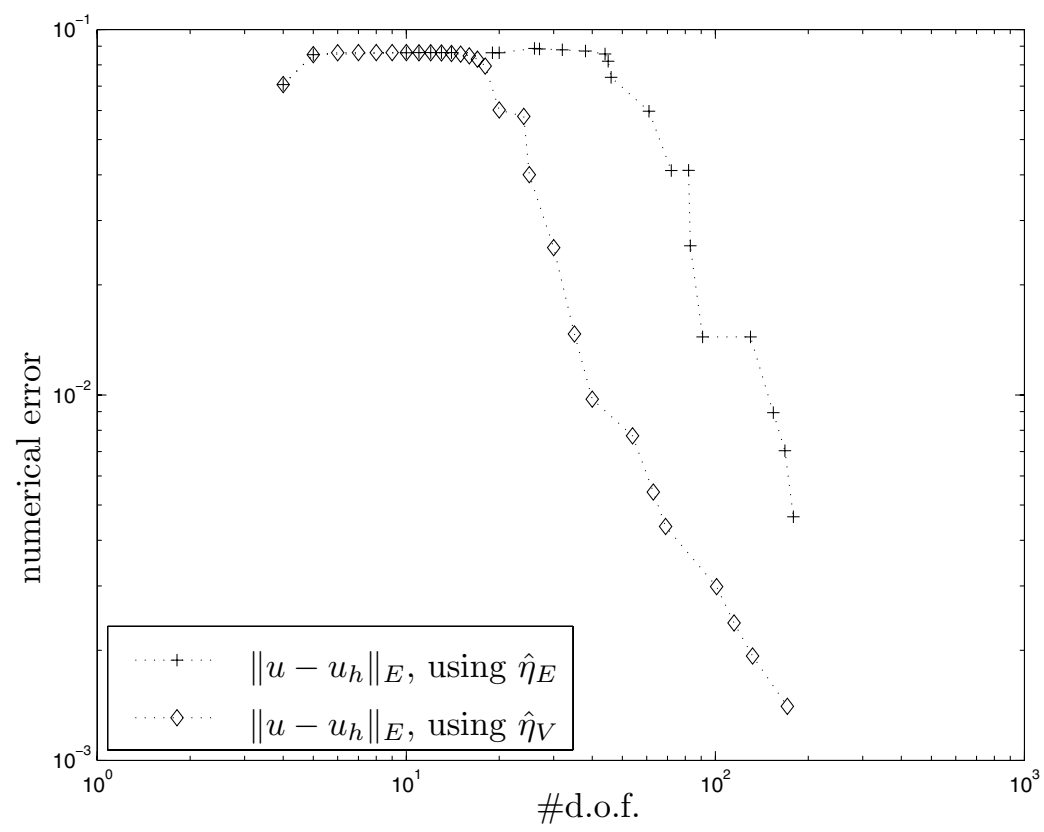

Figure 15. Numerical errors $\left\|u-u_{h}\right\|_{E}$ for problem (AD), $f(x)=$ $\cos (5 / 2 \pi x)$, when using the local estimators (2.93) or (2.94), respectively, for the adaptive refinement

\section{NUMERICAL TESTS IN TWO-DIMENSIONS}

We now consider the two-dimensional model problem

$$
\left\{\begin{aligned}
\mathrm{L} u:=-\varepsilon \Delta u+\beta \cdot \nabla u+\rho u & =f & & \text { in } \Omega, \\
u & =0 & & \text { on } \partial \Omega,
\end{aligned}\right.
$$

where $\Omega$ is the unitary $(L=1)$ square $(0,1)^{2}$. In the next tests, we shall take $\varepsilon>0$, $\beta=\left[\begin{array}{ll}1 & 0\end{array}\right]$, and $\rho=0$. For the sake of simplicity, in the present section we denote 
the differential operator, the estimators, the norms, the mesh by the same notation of 92 , though we refer to new two-dimensional objects.

The previous a-posteriori estimates, which have been rigorously derived in $\$ 2$ for (1.1), can be formally extended in multi-dimensions. One possibility of doing that, perhaps the most obvious, is the following. For the estimator, we set

$$
\begin{aligned}
\hat{\eta}_{V} & :=\left(\sum_{T \in \mathcal{T}_{h}} \hat{\eta}_{V, T}^{2}+\sum_{e \in \mathcal{E}_{h}^{\circ}} \hat{\eta}_{V, e}^{2}\right)^{1 / 2}, \\
\hat{\eta}_{V, T}^{2} & :=\min \left\{\rho^{-1},\|\beta\|^{-1}\left(1+\log ^{+}(P e)\right) h_{T}, \varepsilon^{-1} h_{T}^{2}\right\}\left\|f-\mathrm{L} u_{h}\right\|_{L^{2}(T)}^{2}, \\
\hat{\eta}_{V, e}^{2} & :=\min \left\{\rho^{-1 / 2} \varepsilon^{-1 / 2},\|\beta\|^{-1}\left(1+\log ^{+}(P e)\right), \varepsilon^{-1} h_{e}\right\}\left\|\varepsilon\left[\nabla u_{h} \cdot \mathbf{n}\right]\right\|_{L^{2}(e)}^{2},
\end{aligned}
$$

where $\mathcal{T}_{h}$ now denotes the decomposition of $\Omega$ into triangular elements $T$ of diameter $h_{T}, \mathcal{E}_{h}^{\circ}$ is the set of the internal edges $e$ of length $h_{e},\left[\nabla u_{h} \cdot \mathbf{n}\right]$ is the jump of the normal derivative of the discrete solution $u_{h}$ across a given edge. In the same way we set

$$
\begin{aligned}
\hat{\eta}_{E} & :=\left(\sum_{T \in \mathcal{T}_{h}} \hat{\eta}_{E, T}^{2}+\sum_{e \in \mathcal{E}_{h}^{\circ}} \hat{\eta}_{E, e}^{2}\right)^{1 / 2}, \\
\hat{\eta}_{E, T}^{2} & :=\min \left\{\rho^{-1}, \varepsilon^{-1} h_{T}^{2}\right\}\left\|f-\mathrm{L} u_{h}\right\|_{L^{2}(T)}^{2}, \\
\hat{\eta}_{E, e}^{2} & :=\min \left\{\rho^{-1 / 2} \varepsilon^{-1 / 2}, \varepsilon^{-1} h_{e}\right\}\left\|\varepsilon\left[\nabla u_{h} \cdot \mathbf{n}\right]\right\|_{L^{2}(e)}^{2},
\end{aligned}
$$

which is similar to the definition in 23], though we make the dependence on $\rho$ explicit, as in (2.89) ( $\check{\eta}_{V}$ and $\check{\eta}_{E}$ are not taken into consideration in the next tests). For what concern the norms, $\|\cdot\|_{E}$ is the multi-dimensional analogous of (2.7), while for $\|\cdot\|_{V}$ we set

$$
\|w\|_{V}^{2}:=\|w\|_{E}^{2}+\|\beta\|^{-1}\|\beta \cdot \nabla w\|_{H^{-1 / 2}(\Omega)}^{2}, \quad \forall w \in H_{0}^{1}(\Omega),
$$

where $\|\cdot\|_{H^{-1 / 2}(\Omega)}$ is the dual of $L^{-1 / 2}\|\cdot\|_{L^{2}(\Omega)}+|\cdot|_{H^{1 / 2}(\Omega)}$ (see [13, §12.1]). The definition (3.4) is inspired by (2.22), but it is not the multi-dimensional extension of it, in a strict sens $3^{3}$. The advantage of using (3.4) is that it is not difficult to evaluate $\|\cdot\|_{H^{-1 / 2}}$. In particular, we use FFT on a uniform mesh.

We only consider two test cases. First, we set $\varepsilon=10^{-3}, \beta=\left[\begin{array}{ll}1 & 0\end{array}\right]$ and $\rho=0$, $f(x, y)=2 x \cos (\pi y)$; the exact solution exhibits an exponential layer at the outflow $x=1$ and characteristic layers at $y=0$ and $y=1$. For the second test we have $\varepsilon=10^{-6}$, advection and reaction coefficients as before, $f(x, y)=\pi \cos (\pi x) \sin (\pi y)$, and the solution is now smooth up to the boundary.

In both cases, the finite element approximation $u_{h}$ is given by an SUPG formulation 9] with stabilizing parameter $\tau_{T}:=h_{T} /(2\|\beta\|)=h_{T} / 2$. The meshes are uniform and obtained subdividing $\Omega$ into $2^{i} \times 2^{i}$ squares, which are then cut into two triangles; $u_{h}$ is computed for $i=3, \ldots, 8$, that is, $h=2^{1 / 2} 2^{-3}, \ldots, 2^{1 / 2} 2^{-8}$. As an exact solution, we take the Galerkin approximation on a mesh of $2^{21}$ triangles (i.e., $h=2^{1 / 2} 2^{-10}$ ), which fully resolves the fine scales of $u$.

The effectivity indeces $\hat{\eta}_{V} /\left\|u-u_{h}\right\|_{V}$ and $\hat{\eta}_{E} /\left\|u-u_{h}\right\|_{E}$ are plotted in Figure16 (first case, with layer) and Figure 17 (second case, no layers). The behavior of the

\footnotetext{
${ }^{3}$ The "rigorous" extension of (2.16) in multi-dimensions is also possible (see [19]), and guarantees theoretical properties such as 2.21. However, its practical calculation by 2.92 in the tests we propose is too heavy.
} 


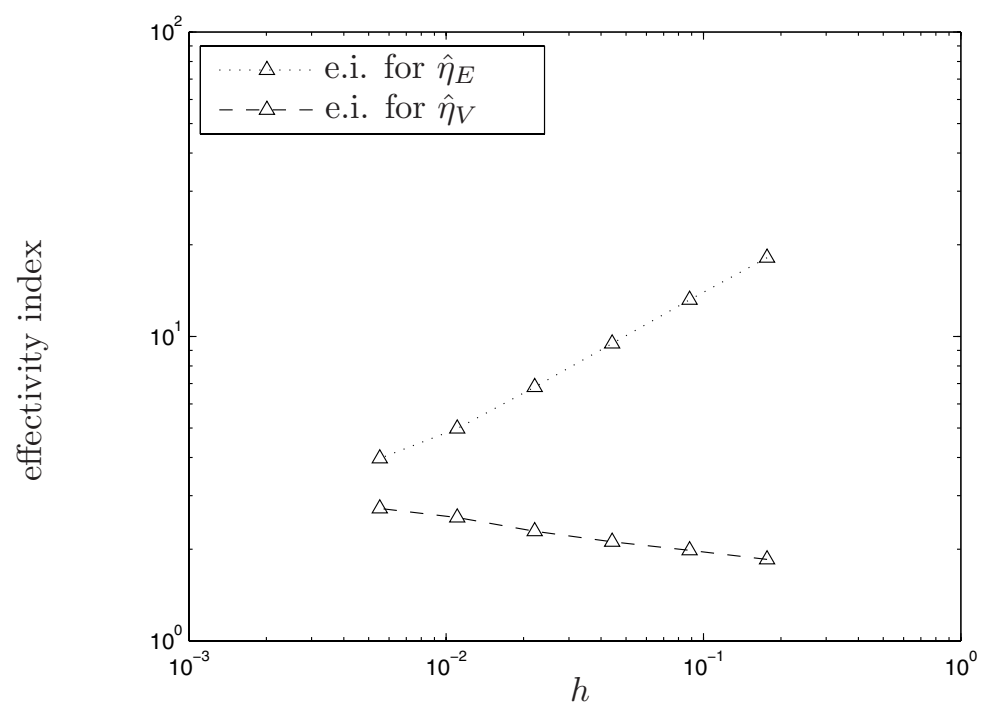

FiguRE 16. Effectivity indeces for the two-dimensional test problem with layers

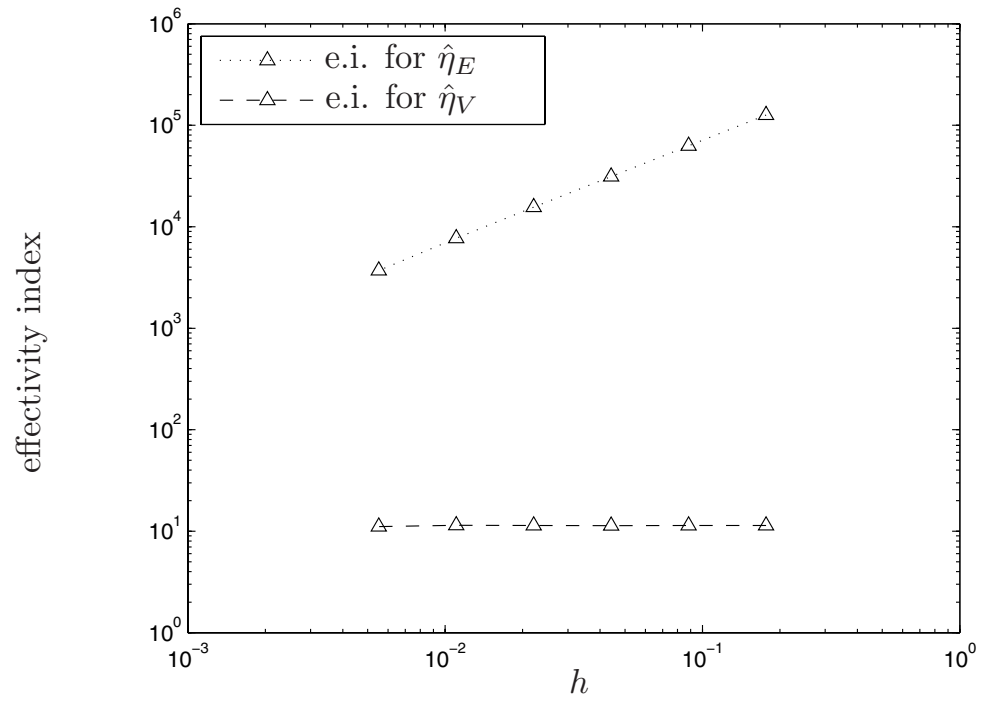

FiguRE 17. Effectivity indeces for the two-dimensional test problem without layers

indeces is similar to the analogous one-dimensional cases. Consider that for Figure 16 we have $\varepsilon=10^{-3}$, and therefore on the finest mesh the regime is moderately advection-dominated, whence the two estimators $\hat{\eta}_{V}$ and $\hat{\eta}_{E}$ are quite similar to each other. Nevertheless, on coarser meshes $\hat{\eta}_{V}$ is superior. This is more clear in Figure 16, where the regime is strongly advection-dominated. 
We tested other cases (not shown) which are analogous to those considered in $\$ 2.5$ and the results are similar.

\section{Conclusion}

In the first part of this work, we have revised the theory of a-posteriori residualbased error estimates (for finite element methods and elliptic problems) and have discussed the results proposed in literature for advection-dominated problems. We have seen that, in order to derive a meaningful estimate, it is important to use an appropriate norm. Our choice of the norm $\|\cdot\|_{V}$ was based on the results of [21].

Then, for the one-dimensional problem, we have obtained upper and lower aposteriori estimators of the numerical error $\left\|u-u_{h}\right\|_{V}$. When the advection is dominant, our upper estimator $\hat{\eta}_{V}$ differs from the lower estimator $\check{\eta}_{V}$ (at most) by the factor $\left(1+\log ^{+}(P e)\right)^{1 / 2}$. There is a weak loss of robustness, and then we refer to the estimate as almost-robust. We have confirmed the theoretical results by numerical tests, showing also that our estimators perform better than the well known ones first proposed in [23].

The theory developed only encompasses the one-dimensional problem. In spite of its simplicity, we have seen that a satisfactory a-posteriori analysis of (1.1) is not a trivial task.

In two dimensions, we only performed a few basic numerical tests, showing that, at least in the examples considered, our estimator seems to preserve the good features of the one-dimensional case, and deserves further investigation.

\section{REFERENCES}

[1] M. Ainsworth and I. Babuška, Reliable and robust a posteriori error estimating for singularly perturbed reaction-diffusion problems, SIAM J. Numer. Anal. 36 (1999), no. 2, 331-353. MR:1668250 (99k:65083)

[2] M. Ainsworth and J. T. Oden, A posteriori error estimation in finite element analysis, Pure and Applied Mathematics (New York), Wiley-Interscience [John Wiley \& Sons], New York, 2000. MR1885308(2003b:65001)

[3] R. Araya, E. Behrens, and R. Rodríguez, An adaptive stabilized finite element scheme for the advection-reaction-diffusion equation, Appl. Numer. Math. 54 (2005), no. 3-4, 491-503. MR2149365 (2006a:65152)

[4] R. Araya, A. H. Poza, and E. P. Stephan, A hierarchical a posteriori error estimate for an advection-diffusion-reaction problem, Math. Models Methods Appl. Sci. 15 (2005), no. 7, 1119-1139. MR2151800 (2006m:65235)

[5] I. Babuška, A. Miller, and M. Vogelius, Adaptive methods and error estimation for elliptic problems of structural mechanics, Adaptive computational methods for partial differential equations (College Park, Md., 1983), SIAM, Philadelphia, PA, 1983, pp. 57-73. MR792521 (87c:65133)

[6] S. Berrone, Robustness in a posteriori error analysis for FEM flow models, Numer. Math. 91 (2002), no. 3, 389-422. MR.1907865 (2003d:76103)

[7] S. Berrone and C. Canuto, Multilevel a posteriori error analysis for reaction-convectiondiffusion problems, Appl. Numer. Math. 50 (2004), no. 3-4, 371-394. MR 2074010 (2005g:65166)

[8] F. Brezzi and A. Russo, Choosing bubbles for advection-diffusion problems, Math. Models Methods Appl. Sci. 4 (1994), no. 4, 571-587. MR1291139(95h:76079)

[9] A. N. Brooks and T. J. R. Hughes, Streamline upwind/Petrov-Galerkin formulations for convection dominated flows with particular emphasis on the incompressible Navier-Stokes equations, Comput. Methods Appl. Mech. Engrg. 32 (1982), no. 1-3, 199-259. MR679322 (83k:76005) 
[10] G. Hauke, M. H. Doweidar, and M. Miana, The multiscale approach to error estimation and adaptivity, Comput. Methods Appl. Mech. Engrg. 195 (2006), no. 13-16, 1573-1593. MR2203982(2006i:76065)

[11] P. Houston, R. Rannacher, and E. Süli, A posteriori error analysis for stabilised finite element approximations of transport problems, Comput. Methods Appl. Mech. Engrg. 190 (2000), no. 11-12, 1483-1508. MR1807010 (2002d:65115)

[12] G. Kunert, A posteriori error estimation for convection dominated problems on anisotropic meshes, Math. Methods Appl. Sci. 26 (2003), no. 7, 589-617. MR1967323 (2004e:65123)

[13] J.-L. Lions and E. Magenes, Non-homogeneous boundary value problems and applications. Vol. I, Springer-Verlag, New York, 1972, Translated from the French by P. Kenneth, Die Grundlehren der mathematischen Wissenschaften, Band 181.

[14] J.-L. Lions and J. Peetre, Sur une classe d'espaces d'interpolation, Inst. Hautes Études Sci. Publ. Math. (1964), no. 19, 5-68. MR0165343 (29:2627)

[15] P. Morin, R. H. Nochetto, and K. G. Siebert, Convergence of adaptive finite element methods, SIAM Rev. 44 (2002), no. 4, 631-658 (2003). MR.1980447

[16] G. Rapin and G. Lube, A stabilized scheme for the Lagrange multiplier method for advection-diffusion equations, Math. Models Methods Appl. Sci. 14 (2004), no. 7, 1035-1060. MR2076484 (2005c:76064)

[17] A. Russo, A posteriori error estimators via bubble functions, Math. Models Methods Appl. Sci. 6 (1996), no. 1, 33-41. MR.1373335 (97e:65112)

[18] G. Sangalli, A robust a posteriori estimator for the residual-free bubbles method applied to advection-diffusion problems, Numer. Math. 89 (2001), no. 2, 379-399. MR1855830 (2002f:65167)

[19] - Construction of a natural norm for the convection-diffusion-reaction operator, Boll. Unione Mat. Ital. Sez. B Artic. Ric. Mat. (8) 7 (2004), no. 2, 336-355. MR2072940 (2005e:35042)

[20] On robust a posteriori estimators for the advection-diffusion-reaction problem, Tech. Report 04-55, ICES Report, 2004.

[21] _ A uniform analysis of nonsymmetric and coercive linear operators, SIAM J. Math. Anal. 36 (2005), no. 6, 2033-2048. MR2178232 (2006g:35037)

[22] H. Triebel, Interpolation theory, function spaces, differential operators, second ed., Johann Ambrosius Barth, Heidelberg, 1995. MR.1328645 (96f:46001)

[23] R. Verfürth, A posteriori error estimators for convection-diffusion equations, Numer. Math. 80 (1998), no. 4, 641-663. MR1650051 (99j:65212)

[24] Robust a posteriori error estimators for a singularly perturbed reaction-diffusion equation, Numer. Math. 78 (1998), no. 3, 479-493. MR1603287 (99c:65218)

[25] , Robust a posteriori error estimates for stationary convection-diffusion equations, SIAM J. Numer. Anal. 43 (2005), no. 4, 1766-1782. MR2182149 (2007d:65116)

[26] M. Vohralík, A posteriori error estimates for lowest-order mixed finite element discretizations of convection-diffusion-reaction equations, to appear in SIAM J. Numer. Anal.

Dipartimento di Matematica, Università di Pavia, Via Ferrata 1, 27100 Pavia, Italy

E-mail address: giancarlo.sangalli@unipv.it 